\title{
EchoGéo
}

\section{L'ancrage au quotidien dans les colonies populaires de la périphérie de Mexico : une approche par les pratiques et les ressources du quartier}

\author{
Jean-François Valette
}

\section{(2) OpenEdition}

Journals

Electronic version

URL: https://journals.openedition.org/echogeo/14837

DOI: $10.4000 /$ echogeo.14837

ISSN: 1963-1197

\section{Publisher}

Pôle de recherche pour l'organisation et la diffusion de l'information géographique (CNRS UMR 8586)

Electronic reference

Jean-François Valette, "L'ancrage au quotidien dans les colonies populaires de la périphérie de Mexico : une approche par les pratiques et les ressources du quartier", EchoGéo [Online], 39 | 2017, Online since 28 March 2017, connection on 10 August 2021. URL: http://journals.openedition.org/ echogeo/14837 ; DOI: https://doi.org/10.4000/echogeo.14837

This text was automatically generated on 10 August 2021.

EchoGéo est mis à disposition selon les termes de la licence Creative Commons Attribution - Pas d'Utilisation Commerciale - Pas de Modification 4.0 International (CC BY-NC-ND) 


\title{
L'ancrage au quotidien dans les colonies populaires de la périphérie de Mexico : une approche par les pratiques et les ressources du quartier
}

\author{
Jean-François Valette
}

\section{AUTHOR'S NOTE}

Ce travail s'est inscrit dans le programme ANR PERISUD 'Dynamiques territoriales à la périphérie des métropoles des Suds", ainsi que dans le programme PICS RESUM "Ressources urbaines et trajectoires familiales dans le Mexique des années 2010", dont les membres sont ici remerciés. Un pré-print en pdf sur lequel les illustrations sont dans un format plus lisible est consultable sur https://halshs.archives-ouvertes.fr/ halshs-01809852

1 Les mobilités articulées aux transformations de l'espace urbain constituent un angle fort pour comprendre les recompositions des villes latino-américaines inscrites dans le processus de métropolisation. Connaissant depuis une trentaine d'années une redistribution de la population selon un modèle de déconcentration-concentration à l'échelle de l'agglomération, la zone métropolitaine de Mexico $(\mathrm{ZMVM})^{1}$ est marquée par un schéma de peuplement où la mobilité intra-urbaine est le principal moteur des dynamiques démographiques. Cette situation, depuis longtemps identifiée dans la plupart des villes, se traduit par la poursuite de l'expansion urbaine, mais également par la densification du bâti existant, particulièrement dans les zones périphériques, les plus dynamiques en termes relatifs (Goüeset et Dureau, 2006 ; Lévy, 2009 ; Dureau, Lulle et al., 2014). 
2 À Mexico, ces périphéries révèlent une grande diversité de formes. La plus répandue dans les milieux modestes - qui composent la majorité de la société mexicaine - reste, depuis les années 1930, la colonie populaire ${ }^{2}$. Il s'agit d'espaces urbains nés d'une production plus large de logements au sein du secteur informel ${ }^{3}$. Dans le paysage urbain, cette réalité se matérialise sous la forme de vastes quartiers populaires ${ }^{4}$ et dans les extensions des villages périphériques intégrés ou non au reste de la ville; leurs habitants appartiennent majoritairement, mais pas exclusivement, aux catégories pauvres (Ribardière et Valette, 2014).

3 Ces vingt dernières années, les colonies populaires ont tendance à connaître un processus de vieillissement de la population et de diffusion de l'accès à l'éducation, pour ne retenir que ces critères, cette évolution allant dans le sens d'un rattrapage, déjà ancien de plus d'un demi-siècle, par rapport au profil moyen de la ville (Ribardière et Valette, 2013). La banalisation du profil social des lieux et de l'accès à la ville des individus renvoie aux trajectoires de normalisation (Lévy, 2009), voire de maturation des contextes résidentiels périphériques (Dureau et Gouëset, 2010 ; Imbert et al., 2011 ; Lessault et Imbert, 2013 ; Berger et al., 2014). Le concept de maturation englobe ainsi la diversification de la composition des populations résidentes, la consolidation du quartier, l'ancrage résidentiel des habitants et le développement des ressources urbaines dans la proximité - les ressources étant ici entendues comme l'ensemble des moyens matériels ou immatériels, économiques ou sociaux disponibles à l'échelle de l'individu ou du groupe, dont les habitants disposent pour assurer leur existence et leur maintien dans le contexte résidentiel considéré. Au total, la maturation se comprend à travers l'articulation entre d'une part, les dynamiques sociodémographiques, morphologiques, économiques et politico-juridiques qui s'observent au niveau des lieux, et d'autre part, les mobilités et ancrages des individus qui résident dans ces lieux (Valette, 2014). Pour comprendre cette maturation des colonies populaires, il est nécessaire d'observer la manière dont les habitants résidant dans ces quartiers utilisent l'espace du point de vue des mobilités, et de s'intéresser à la manière dont ils vivent, déménagent, se projettent dans des espaces pourtant nés dans l'illégalité.

4 Notre propos reposera sur l'analyse de l'expérience qu'ont les habitants des périphéries, expérience qui sous-entend une profondeur historique marquée de la mobilité résidentielle comme quotidienne. L'hypothèse de l'expérience ici explorée est celle d'un ensemble de lieux utilisés dans une pratique résidentielle - le système résidentiel (Le Bris, 1981; Dureau, 2002)-, mais aussi sociale, économique et symbolique au sein de l'espace de vie, qui ne se limite ni au lieu de résidence ni à l'espace des activités (Lelièvre et Robette, 2006). L'expérience dans un quartier souvent perçu comme par défaut pourrait ainsi relever de choix et de compétences particulières, notamment pour habiter, voisiner, explorer et circuler.

5 L'expérience que fait une personne d'un lieu ou des lieux qu'elle fréquente introduit la notion d'ancrage (Imbert, 2005, p. 5). L'ancrage, social et résidentiel, interroge les circulations des individus et de leur entourage dans les différentes échelles spatiales et temporelles de l'espace de vie. Cette notion se pose comme grille de lecture des comportements sociaux et spatiaux des individus, dans la recherche des ressources de la proximité , mais aussi dans la constitution de réseaux au sein d'un espace plus large, connectant plusieurs lieux d'ancrage, dans la ville et au-delà (Imbert, 2005 ; Brevet, 2008 ; Bertrand, 2010a, 2010b; Pujol, 2010 ; Contreras, 2012). L'ancrage est ainsi une dynamique multidimensionnelle et multiscalaire devant rendre compte, tout à la fois 
des pratiques résidentielles témoignant d'une insertion urbaine (liens, mobilité, consolidation, investissement, évolution démographique des ménages), des stratégies immobilières et d'accès au sol, et de la participation politique et organisationnelle autour de l'insertion durable dans des réseaux sociaux (Bertrand, 2011). L'ensemble de ces dimensions prend sens dans le contexte urbain mexicain étudié ici, où pratiques résidentielles de proximité et dynamiques foncières et participatives - clefs de compréhension d'une relative stabilité résidentielle et d'une organisation urbaine de proximité - font écho aux rapports de pouvoir au cœur de l'évolution des colonies populaires.

6 L'approche des ancrages combine l'analyse des mobilités résidentielles et celle des mobilités quotidiennes ${ }^{6}$. Trancher entre ces deux catégories de mobilités implique des choix difficiles, d'autant qu'il ne s'agit pas de les opposer, voire de les séparer aussi abruptement, sur une seule question d'échelle temporelle. Toutefois, l'analyse spécifique des mobilités quotidiennes que nous proposons ici, même si elle ne couvre qu'une partie de l'étude des dynamiques urbaines par les mobilités spatiales, permet une approche de la structuration des espaces de vie des individus et de leur entourage, ainsi que des changements urbains à l'œuvre (Dureau et al., 2014, p. 81). L'observation des mobilités quotidiennes autour de la résidence en colonie populaire périphérique répond ainsi à une volonté de compréhension fine des dynamiques d'ancrage. Ce sont alors les systèmes d'activités des habitants qui sont interrogés, au sens où l'on observe la « manière dont les individus affirment répartir leurs activités entre chacun des lieux qui structurent leur espace de circulation » (Dubucs et Giroud, 2014, p. 366).

7 L'objectif ici est double: comprendre quelques schémas de mobilités quotidiennes d'habitants d'une part; identifier par-là des variables des mécanismes d'apprivoisement de leur contexte résidentiel d'autre part - dans le sens où les habitants de ces espaces initialement illégaux doivent déployer de nombreux efforts pour rendre familier, domestique, un contexte difficile au départ. Une telle approche entend rendre compte des processus socialement et spatialement différenciés d'intégration progressive à la ville par le jeu des mobilités - intégration qui inscrit le contexte local dans des réinventions ${ }^{7}$ et des reconfigurations, notamment foncières et politiques.

8 Afin d'étudier les mécanismes et les implications de ces ancrages au quotidien dans les colonies populaires périphériques de Mexico, nous décomposons notre propos en quatre temps. Premièrement, les enjeux de la recherche et la méthodologie sont exposés. Pour les trois temps suivants, au sein de quatre quartiers périphériques témoins, et en variant les niveaux de contextualisation, trois points de vue complémentaires sont investis, renvoyant chacun à une échelle d'analyse spécifique : i) les périphéries dans l'espace des pratiques quotidiennes, ii) les périphéries comme espace de ressources, et iii) les périphéries comme point de départ des ancrages urbains. D'abord, il s'agit de mettre en avant les liens au quartier périphérique du point de vue des pratiques - les fréquentations habituelles d'un lieu, « ce que l'humanité, les sociétés, les individus font tous les jours, et qui fait le monde, dont l'espace géographique " (Brunet et al., 1992, p. 360). Ensuite, on explore, du point de vue des lieux pratiqués ou potentiellement fréquentés localement, la fonctionnalisation économique et sociale du quartier. Enfin, du point de vue des individus, le quartier «ne suffit pas »: les mobilités quotidiennes témoignent de la présence forte de ressources urbaines dans l'espace local, mais aussi d'une relative intensité des déplacements à 
d'autres échelles, allant dans le sens d'une intégration au reste de la ville. Le croisement des différentes pratiques des espaces locaux et métropolitains autorise la mise au jour de plusieurs types de rapports au quartier et d'une diversité des ancrages qui s'inscrivent dans ces lieux.

\section{Pour une approche raisonnée de l'ancrage au quotidien}

9 L'approche en miroir des ancrages à l'aune des mobilités quotidiennes et des ressources urbaines par le suivi des pratiques et des systèmes d'activités permet une lecture des dynamiques contemporaines des périphéries populaires de Mexico. Les enjeux soulevés par cette approche - en termes de rapport à la centralité et d'intégration - doivent être explicités. Puis les sources et méthodes mobilisées pour traiter ces dynamiques seront présentées, ainsi que les terrains d'étude dans lesquels s'est inscrit le travail empirique.

\section{Éclairer les ancrages périphériques et les ressources urbaines par les mobilités quotidiennes}

10 L'examen des ancrages dans les périphéries de Mexico passe par l'observation des usages urbains - usages qui mettent à leur tour en lumière les ressources urbaines autour de la résidence. Sans prétendre à une analyse exhaustive de ces ressources, il s'agit de souligner leur présence et les mécanismes de leur développement dans le contexte local de la résidence en colonie populaire et de contribuer à leur appréhension comme des indicateurs des inégalités socio-spatiales à un niveau fin (Ribardière, 2017).

$11 \mathrm{Au}$ carrefour entre regard fonctionnaliste sur l'urbain et prise en compte des expériences vécues des habitants, la question des déplacements des individus nourrit aussi en retour l'étude des ressources urbaines et des inégalités dans leur accès. Outre un regard sur les réseaux de circulation qui structurent la ville, l'entrée par les mobilités permet une lecture transversale et longitudinale des phénomènes de différenciation de l'espace urbain, le caractère distinctif de la mobilité étant largement démontré. Dans les périphéries urbaines, on observe une densification et un renforcement du fonctionnement interne - émergence de bassins de vie ${ }^{8}$ ou encore reproduction des inégalités - reposant sur un système de mobilités de proximité. Si ces phénomènes sont observés dans la région parisienne (Lévy, 2009; Berger et al., 2014 ; Jouffe et al., 2015 ; Berroir et al., 2016), la question se pose également pour l'analyse des espaces périphériques urbains à partir des expériences du Sud comme à Mexico. Observe-t-on ici aussi, à la lecture des mobilités, une relative autonomisation par rapport aux centres ${ }^{9}$ ?

12 Dans le cadre de cet article, s'il n'est pas question de négliger l'importance de considérer et d'articuler les deux types de mobilité, résidentielle et quotidienne, pour appréhender les ancrages, l'attention est plus particulièrement portée sur le volet quotidien de l'expérience urbaine. Suivre ces déplacements quotidiens permet l'identification des marquages sociaux des espaces publics, et une réflexion sur les systèmes de lieux, les territoires urbains dessinés par l'approche intégrée de ces mobilités et les inégalités qu'elles sous-tendent (Dureau et al., 2014 ; Gouëset et al., 2014 ; Dureau et al., 2014 ; Jouffe et al., 2015). 
13 À l'échelle de la ville, la territorialisation des logiques de production urbaine (logements, services et emplois entre autres) détermine les conditions d'accès aux ressources spatialisées, et par là, les schémas de mobilités urbaines. Le lien fort entre la mobilité quotidienne et la ségrégation sociale est ainsi souligné à Mexico, notamment pour la périphérie, d'une part en raison d'un usage différencié de l'espace et d'une accessibilité moindre aux transports selon la zone et la catégorie socio-économique (Salazar, 2010), d'autre part en raison de la localisation même des ressources en termes d'emplois et de services (Suárez et al., 2015 ; Ruiz Rivera et al., 2016). La segmentation des pratiques urbaines que la prégnance des logiques privées - à l'origine de la localisation des ressources - implique à l'échelle de la ville, surtout en périphérie, est liée à la polarisation des catégories supérieures et moyennes-supérieures dans l'ouest de la métropole (Duhau, 2003 ; Guerrien, 2004) (illustration 2). Mais l'exacerbation des ségrégations socio-spatiales lues par l'accès à la ville reste à nuancer, notamment pour les quartiers pauvres, du fait d'un côté, de la souplesse et de l'efficacité sociale du système de transports en commun de Mexico pour les catégories modestes, et de l'autre, de l'importance des pratiques de proximités en périphérie en raison de la densité des activités économiques informelles qui prennent place dans ces espaces (Guerrien, 2002 ; Paquette, 2010 ; Suárez et al., 2015). La mise en avant des pratiques commerciales et de la recherche de ressources locales, combinée à l'accès à la mobilité quotidienne à Mexico, donne une lecture nouvelle du lien des espaces populaires périphériques au reste de la ville. Elle remet notamment en cause les schémas selon lesquels les périphéries populaires concentreraient uniquement des situations de dépendance vis-à-vis de la mobilité vers le centre, ou encore des situations de relégation-immobilité. Les pratiques locales et les formes d'ancrage des individus y sont introduites et permettent l'approche de l'accès à la ville aussi en termes d'intégration plutôt que de seule exclusion.

Le lien ambivalent entre la recherche de ressources dans le local et la tendance plus globale à la segmentation des pratiques urbaines dans l'ensemble de la ville doit alors être interrogé. Comprendre les processus et les modèles généraux des systèmes de mobilité dans les périphéries populaires de Mexico renvoie à appréhender les trajectoires individuelles et contextuelles s'inscrivant dans ces espaces.

\section{Sources et méthodes. Étude dans quatre quartiers périphériques}

15 Au sein des périphéries de Mexico, la comparaison a porté sur un ensemble de quartiers populaires en cours de consolidation, construits depuis les années 1990 et marqués par une relative croissance démographique liée à la migration intra-urbaine. L'objectif de cette sélection n'était pas la représentativité métropolitaine, mais plutôt la prise en compte d'une diversité des situations possibles, afin de couvrir une palette variée de réalités périphériques, en termes de connexion avec le reste de la ville, de morphologie et d'environnement urbains, et de contexte politique et administratif. Ainsi, quatre groupes de quartiers (d'environ 15000 habitants chacun) dans quatre municipalités ont été retenus (Illustrations 1 et 2). 
Milpa Alta, au sud, est la délégation la moins urbanisée de la Ciudad de México (DF), dans un contexte de villages montagnards périurbains mal connectés au reste de la ville, à 3 heures de transport du Zócalo - la place de la Constitution, symbolisant le centre historique, politique et économique de la ville, centre où restent encore concentrés les activités et les emplois. Plus précisément, le village de San Pablo Oztotepec a été étudié : sa croissance radiale s'est principalement réalisée autour du noyau villageois, sur des terrains au régime foncier spécifique, où la construction est interdite. Les profils socio-spatiaux des îlots, appréhendés selon une entrée par la pauvreté dans d'autres travaux (Ribardière, Valette, 2014; Valette, 2015), renvoient à un gradient radioconcentrique de précarité.

La délégation Tláhuac, en périphérie sud-est du DF, est beaucoup plus densément peuplée et reste dynamique démographiquement (+1.2\%/an depuis 2000). La zone spécifique de Santiago Zapotitlán a connu une spéculation foncière élevée, avec l'arrivée en 2015 d'une nouvelle ligne de métro. Le gradient d'urbanisation nord-sud est net : à partir des années 1970, les terrains agricoles de l'ejido au sud du village sont urbanisés, prioritairement par les « enfants du village », dans la zone de La Conchita. Ce mouvement de peuplement se poursuit, jusqu'à la colonie Ampliación La Conchita, et est couplé, à Tempiluli, par la densification progressive des terrains situés entre le centre de Tláhuac et Zapotitlán. Les contextes résidentiels les plus récents sont particulièrement marqués par la pauvreté.

Dans l'État de Mexico, le municipe de La Paz, connecté au reste de la métropole par le métro, a vu sa population doubler en 20 ans (près de 300000 habitants en 2015), principalement du fait de la mobilité résidentielle intra-urbaine. Sur les collines de l'est, à Jorge Jiménez Cantú et Lomas San Sebastián, le paysage est marqué par l'extension de colonies populaires le long d'un vaste front d'urbanisation, nourri par le desserrement des zones péricentrales de Nezahualcóyotl et Iztapalapa, dans un contexte de précarité forte et généralisée.

Enfin, Cuautitlán, également dans l'État de Mexico, renvoie aux zones de desserrement des activités industrielles du nord de Mexico depuis 30 ans, et à un des espaces de la croissance urbaine massive sous la forme de grands ensembles de logements sociaux en accès à la propriété aidé. La prégnance de ces « quartiers populaires légaux » est forte : plus de $60 \%$ des habitants (près de 86000 personnes) résident dans les différentes générations de logements sociaux. Depuis 2008 , la municipalité est bien reliée au centre-ville grâce au Suburbano (train de banlieue). L'extension en colonies populaires (Lázaro Cárdenas, Tlaxculpas, Tlaltepan) se fait dans les interstices laissés entre les lotissements légaux: si les contextes de pauvreté n'y sont pas absents, les profils sont là moins marqués que dans les trois autres terrains d'étude.

Illustration 2 - Peuplement et profils socio-spatiaux de quatre quartiers périphériques

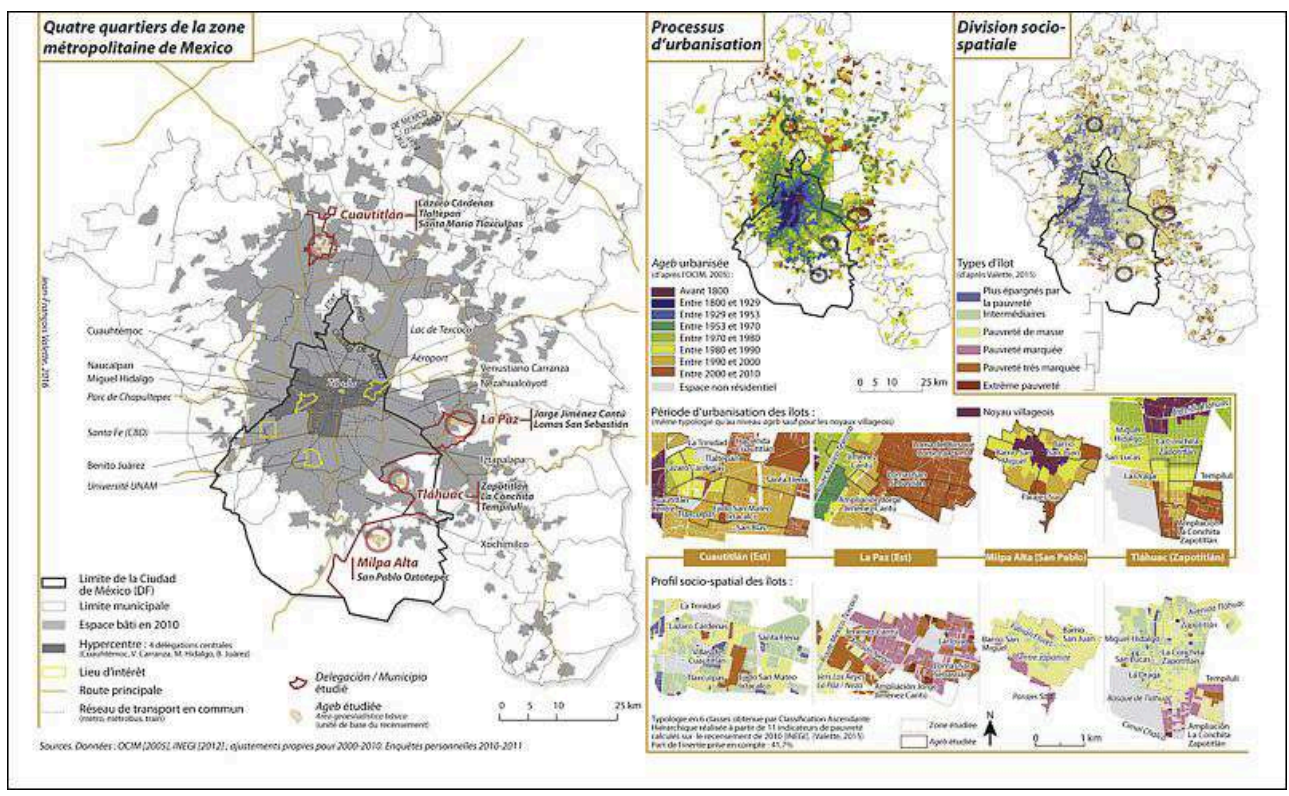

16 Dans ces quatre quartiers, il s'est agi d'analyser simultanément différents types de données, afin de croiser les informations et de varier les niveaux de contextualisation : de l'individu à l'espace métropolitain, en passant par le ménage, la maison, l'îlot, le quartier et la municipalité. Des sources systématiques et précises disponibles sur Mexico ont ainsi été mobilisées et enrichies par des explorations empiriques. Ainsi, premièrement, les recensements de population effectués par l'INEGI (Institut national des statistiques et de la géographie), notamment celui de 2010, aux niveaux 
détaillés (muestra) et agrégés (censo), offrent une lecture des matrices de flux domiciletravail, mais aussi des caractéristiques sociales des contextes résidentiels et des trajectoires des individus. Deuxièmement, l'enquête de mobilité quotidienne OrigineDestination de 2007 (EOD) (INEGI, 2007) ${ }^{10}$ a aussi été exploitée, permettant l'analyse des motivations, modes, coûts, temps et localisations des déplacements. Pour ces deux sources, la géolocalisation n'est pas très précise : municipalité pour le recensement ${ }^{11}$, district pour l'EOD ${ }^{12}$. Enfin, troisièmement, les recensements économiques de 2009 et 2014, ainsi que l'enquête DENUE (Répertoire statistique national des unités économiques) de 2012, ont permis d'identifier et d'inventorier les activités économiques dans les quartiers étudiés, et d'approcher finement les fonctions urbaines et l'existence de ressources à travers le tissu local ${ }^{13}$ de commerces et de services.

Ensuite, afin de mieux cerner les pratiques spatiales des habitants et leur rapport à la ville, deux séries d'enquêtes de terrain, purement exploratoires ${ }^{14}$, à l'aide d'entretiens et de questionnaires au niveau des individus résidents et de leur entourage, ont été menées ${ }^{15}$. Premièrement, une série de 139 entretiens longs semi-directifs, parfois répétés, dans chacun des terrains d'étude a visé la compréhension des parcours de vie et les perceptions et expériences du quartier et de la ville (enquête E1). Les questionnements rejoignent là ceux déjà éprouvés lors de grandes enquêtes relatives à l'approche biographique (GRAB, 1999 ; Dureau et al., 2006 ; Bertrand, 2001), à la mobilité (Imbert et al., 2014 ; Dureau et al., 2014), et aux rapports aux quartiers ${ }^{16}$ (Authier, 2001). Plusieurs profils de résidents ont été sondés - responsables locaux, groupes politiques, commerçants, anciens installés, et familles rencontrées de manière aléatoire - sur leur situation actuelle, leur itinéraire résidentiel, leur entourage, leurs relations à l'espace et leurs projets de vie. Deuxièmement, une série de 366 questionnaires courts (enquête E2) auprès des jeunes parents des quartiers étudiés (diffusion par le canal des groupes scolaires) a permis d'appréhender, spécifiquement pour une génération d'habitants en cours de stabilisation résidentielle, les contours de l'espace de vie pratiques de l'espace urbain, lieux et statuts de résidence, entourage, perception du lieu -, quelques mécanismes d'appropriation de l'espace local, ainsi que la manière de se projeter et d'envisager les stratégies résidentielles futures.

L'articulation de ces différents matériaux et niveaux d'observation répond à une volonté de pallier les faiblesses propres à chaque source en les croisant. Outre la possibilité de monter en généralités d'un côté, et celle de prendre en compte des dimensions fines de l'autre, l'utilisation simultanée de sources systématiques et empiriques exploratoires permet un éclairage pluriel sur les pratiques quotidiennes et les systèmes d'activités des résidents des quartiers populaires périphériques.

\section{Le suivi des pratiques : les ressources dans le local}

À l'échelle de la métropole, la centralité des pouvoirs et des activités a toujours été particulièrement forte: plus de la moitié des flux domicile-travail sont toujours polarisés dans les quatre délégations centrales: Cuauhtémoc, Benito Juárez, Miguel Hidalgo et Venustiano Carranza (INEGI, 2007). Cette polarisation s'est même renforcée, malgré le déclin démographique du centre (Salazar et Sobrino, 2006). Pourtant, outre ce poids du centre, nous faisons l'hypothèse que la fonctionnalisation des périphéries et les schémas de peuplement centrifuges ont contribué en parallèle à une augmentation des liens intra-périphériques. Ces liens sont-ils le résultat d'une captivité ou d'une 
complexification des systèmes d'activités des résidents? D'abord, les expériences individuelles et collectives peuvent être observées du point de vue du lieu, à partir du point d'ancrage qu'est le contexte résidentiel en périphéries populaires.

\section{L'ancrage dans le quartier n'est pas synonyme d'enclavement}

Dans un contexte urbain où la mobilité des pauvres est souvent érigée comme plus difficile qu'ailleurs dans la ville et où la mobilité généralisée renforcerait les inégalités socio-spatiales (Bacqué et Fol, 2007 ; Fol, 2009, 2010 ; Suárez et al., 2015), une première approche des manières d'habiter le quartier peut consister à mesurer son négatif, à savoir la pratique de lieux extérieurs au quartier. Au cours d'un travail empirique auprès d'un échantillon d'habitants (E1) auxquels il était demandé la localisation de leurs activités, les fréquences de sortie du quartier des enquêtés ont été explorées à travers les questions suivantes : "vous arrive-t-il de sortir du quartier? Si oui, à quelle fréquence, pour quels motifs principaux (ceux qui sont récurrents), pour aller où le plus souvent? " (illustration 3). Ainsi, quasiment toutes les personnes enquêtées dont les réponses étaient exploitables sur ce sujet (106/109) sortent de leur quartier de résidence au moins une fois par semaine et plus de la moitié en sortent quotidiennement. Près de la moitié des déplacements hors quartier renvoient à un motif de travail. Du point de vue spatial, la relation classique entre la distance et la fréquence se retrouve, les déplacements réguliers étant majoritairement ceux s'opérant dans une proximité relative.

Illustration 3 - Fréquence et motif de sortie du quartier

\begin{tabular}{|c|c|c|c|}
\hline Fréquence de sortie du quartier & Total & Motif récurrent de déplacement en dehors du quartier & Total \\
\hline Moins d'une fois par semaine & 3 & Travail & 46 \\
\hline Une fois par semaine & 20 & Réseau amical & 14 \\
\hline Plusieurs fois par semaine & 9 & Démarches administratives et politiques & 13 \\
\hline Une fois par jour & 21 & Famille & 7 \\
\hline Plusieurs fois par jour & 56 & Établissement scolaire & 6 \\
\hline Pas de réponse & 30 & Autre résidence (multirésidence) & 5 \\
\hline \multirow[t]{5}{*}{ I Iotal des reponses exploitadies } & 109 & Religion & 4 \\
\hline & & Courses & 4 \\
\hline & & Récréation & 3 \\
\hline & & Pas de réponse & 51 \\
\hline & & Total des réponses exploitables & 88 \\
\hline
\end{tabular}

À un échelon moins fin - celui du district -, une analyse à partir de données systématiques fournies par l'enquête EOD de 2007 est possible (illustration 5). Selon cette enquête, on estime à plus de 22 millions le nombre de voyages quotidiens dans la ZMVM. L'observation de tous les trajets effectués au départ et à destination (quels que soient le motif et le mode de déplacement ${ }^{17}$ ) vers et depuis les 4 districts recouvrant les quartiers étudiés permet de mettre en avant le fait que les espaces concernés par ces mobilités dépassent largement la proximité relative à cet échelon du district. Un gradient est lisible autour de chaque zone, couplé à une sorte d'entonnoir orienté vers le centre de la métropole.

L'analyse des sorties régulières du quartier témoigne donc sans surprise d'une articulation des systèmes d'activités (travail, études, courses, loisirs, visite d'un tiers, démarches administratives, etc.) dans les autres espaces de la ville (centres, souscentres, autres quartiers périphériques) et de la structuration des espaces de vie des individus bien au-delà des seules limites du local - défini à l'échelon du district comme 
à celui du quartier de résidence. L'approche de l'ancrage dans ces quartiers populaires s'avère ainsi affranchie d'une focale sur la seule sédentarité et d'un regard unique sur l'enclavement. L'ancrage immobile des individus et des ménages, s'il est mis au jour, doit alors être analysé au regard d'une part, de l'insertion différenciée dans le marché de l'emploi, d'autre part, des effets de séparation physique (infrastructure-barrière, éloignement) et symbolique liés à la géographie spécifique des contextes résidentiels (illustration 4) (Motte-Baumvol et al., 2016).

\section{Illustration 4 - Perception de l'enclavement par deux habitants de l'est de La Paz}

Un échange au sein d'un jeune couple de Jiménez Cantú à La Paz illustre à la fois la diversité des pratiques de mobilités, autant que des distinctions qui existent entre les individus - que les différences soient effectives et/ou représentées. Ainsi, en 2010, Lupe, 20 ans, et Benjamin, 23 ans, déclarent au sujet de leurs déplacements dans le reste de la ville de Mexico:

Benjamin (serveur à temps partiel) : « Oui, la plupart du temps, je ne travaille pas dans le quartier, je vais un peu partout. Ça dépend beaucoup des opportunités »

Lupe (avec son fils sur les genoux) : «Nous deux, on ne sort jamais d'ici! »

B. : « C'est qu'il n'y a rien à faire ici! »

L. «Mais non, ce n'est pas vrai, c'est juste qu'ici, c'est plus tranquille».

Si le mari évoque sa mobilité relativement importante au sein de l'espace métropolitain, la représentation du quartier de résidence s'en trouve a priori particulièrement dépréciée, pointant le manque d'activités et d'opportunités d'emplois. L'approche est très différenciée selon le genre et la position socio-professionnelle au sein de ce couple. Lupe témoigne d'un regard contraire, à la fois sur la mobilité et sur son contexte résidentiel. Elle semble évoquer avec un certain fatalisme sa faible mobilité quotidienne dans la ville, mais son discours est moins catégorique au sujet des représentations du local : elle souligne l'attachement qu'elle ressent au sujet de son quartier et l'inscription historique personnelle qu'elle y projette.

Illustration 5 - Les espaces de mobilité quotidienne de quatre districts périphériques

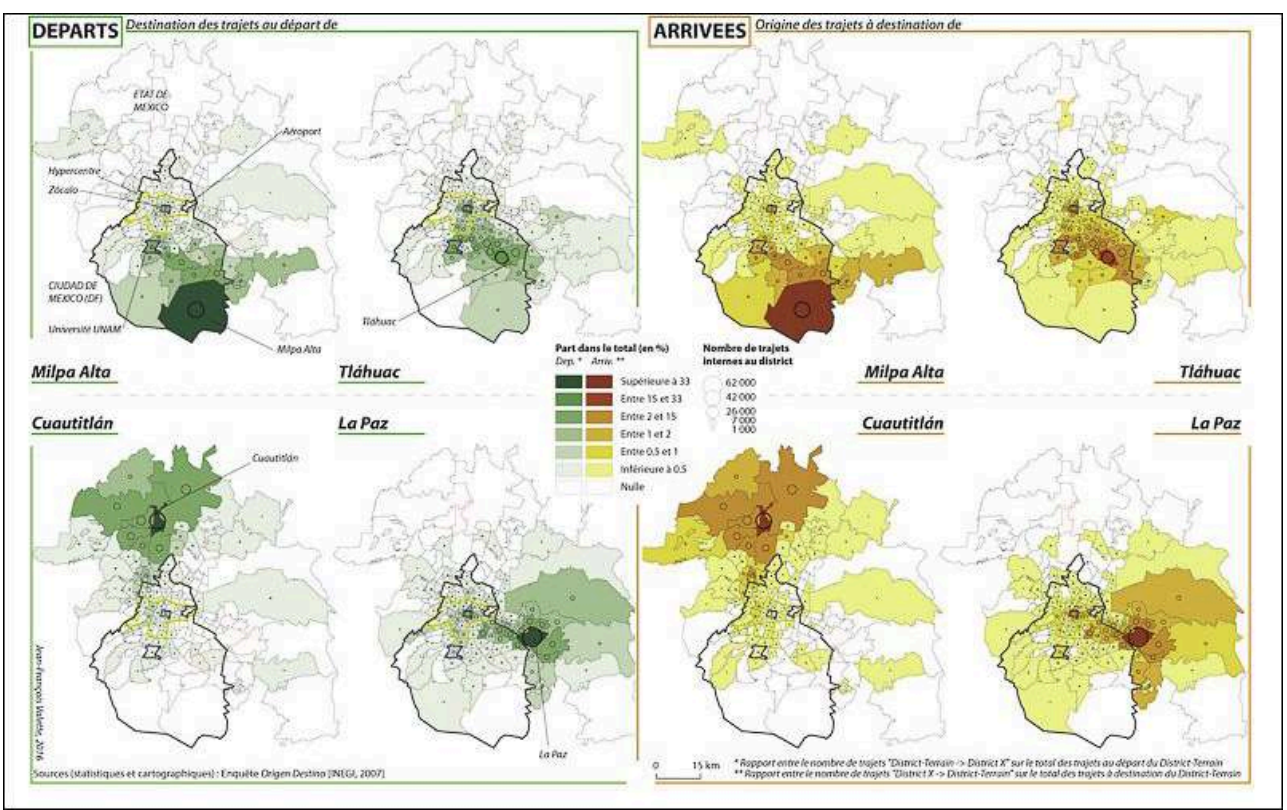

Plus qu'une hantise de l'immobilité ${ }^{18}$ et sans pour autant nier l'existence incontestable de véritables captifs dans les colonies populaires, les ancrages territoriaux en formation dans les quartiers périphériques étudiés ne semblent pas enfermer les 
habitants interrogés. Ils mettent néanmoins en lumière une forte inscription spatiale dans la proximité.

\section{La force de la proximité}

Afin de cerner les ressources dans le contexte résidentiel, les pratiques sont lues du point de vue des motifs de déplacement. En partant de questionnements classiques sur les mobilités quotidiennes dans les quartiers populaires latino-américains (Baby-Collin, 2005; Gouëset et al., 2014), il s'est agi d'observer la localisation de l'activité professionnelle, l'accès aux services (école, santé), les pratiques commerciales et récréatives et les autres sociabilités.

\section{Le lien domicile-travail : un fonctionnement interne remarquable}

Une exploration des pratiques de l'espace citadin de l'activité professionnelle est possible grâce aux données disponibles dans l'échantillon détaillé du recensement de 2010 : en croisant le municipe où s'exerce l'activité professionnelle et le municipe de résidence, on obtient une image des mobilités domicile-travail (illustration 6).

La thèse de l'ancrage local - au sens de municipal ici - de l'emploi est confirmée. Dans l'ensemble de la ville, cet ancrage est relativement élevé : en moyenne dans la ZMVM, $57 \%$ des actifs travaillent dans leur municipe de résidence. Les habitants des quatre municipes étudiés vont travailler en majorité dans leur propre municipe de résidence : $64 \%$ des actifs de Milpa Alta, $55 \%$ de Tláhuac et $52 \%$ de La Paz. C'est seulement à Cuautitlán que la tendance est moindre ( $40 \%$ ), en raison de la polarisation des zones d'activités (industrielles notamment) de Cuautitlán Izcalli et Tultitlán. Les autres zones de travail sont les municipes voisins, offrant des espaces de centralité secondaire: Xochimilco pour Milpa Alta, Iztapalapa pour Tláhuac et La Paz.

L'observation des zones de recrutement des municipes étudiés renvoie au même constat. De ce point de vue (des arrivées), l'ancrage périphérique semble plus affirmé qu'à l'échelle métropolitaine où, en moyenne en 2010, $62 \%$ des travailleurs d'une municipalité y sont aussi résidents. On observe un fonctionnement interne - avec plus de $75 \%$ des travailleurs résidant dans le municipe - pour Tláhuac, La Paz et Milpa Alta, et plus diffus - bien que toujours de grande proximité (municipale) - à Cuautitlán. En dehors de cet endoréisme, ce sont les municipes adjacents - aussi bien péricentraux que plus périphériques - qui constituent les espaces résidentiels des travailleurs des espaces étudiés. Ces municipalités, marquées par des concentrations de pauvreté et par le poids dominant de l'emploi informel ${ }^{19}$, seraient pourtant les lieux d'un commuting globalement moins fort que ne le laisserait supposer la structure métropolitaine du marché de l'emploi formel (Suárez et al., 2015). 


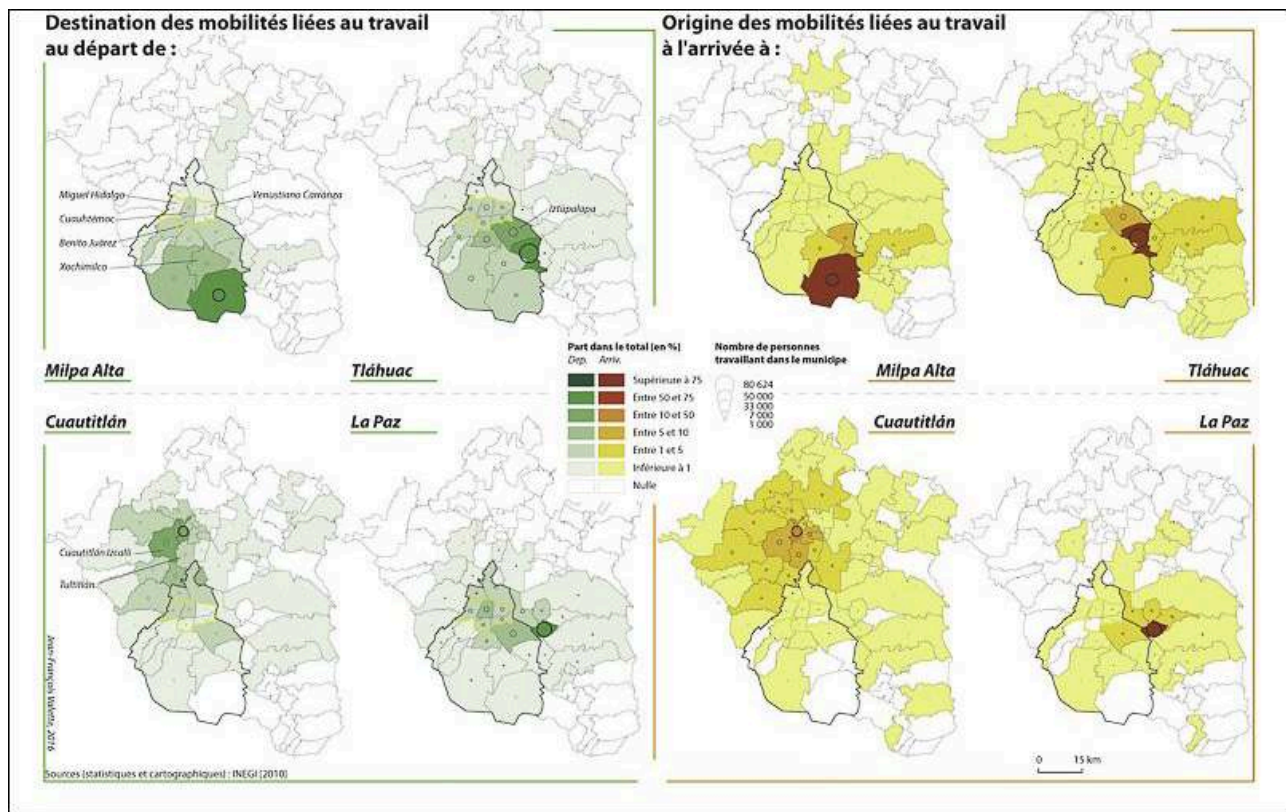

Au-delà des niveaux agrégés, le regard sur de petits échantillons appréhendés empiriquement met en évidence des schémas plus fins articulant deux échelles. En plus de la polarisation des emplois par les axes de transports et les bassins centraux et péricentraux traditionnels, la force de la proximité est une nouvelle fois soulignée. Loin de représenter uniquement des banlieues-dortoirs, les quartiers populaires périphériques de Mexico sont aussi des zones d'activités économiques importantes. À la question " où travaillez-vous en général ? (139 entretiens de l'enquête E1) ou « où se localise l'activité professionnelle principale du chef de ménage? » (366 questionnaires de l'enquête E2), plus des deux tiers des personnes interrogées renvoient à une localisation interne au municipe de résidence. Apparaissent ainsi des stratégies élaborées par rapport à un cadre d'accessibilité au reste de la ville relativement limité, du fait des coûts de la mobilité d'un côté, du niveau d'éducation - moins on est qualifié, plus on privilégie un marché de l'emploi local, la compétitivité étant moindre à petite échelle - de l'autre.

D'une manière générale, les flux de mobilités quotidiennes liés à l'emploi, au départ ou à destination des espaces périphériques étudiés, révèlent un lien double avec le reste de la ville : la dépendance au centre n'est pas remise en question, mais les dynamiques internes et celles de proximité sont fortes. La deuxième couronne dans laquelle s'inscrivent les quatre quartiers étudiés joue un rôle de pivot, lié au centre - plus particulièrement au péricentre et à la première couronne--, et polarisant des périphéries plus éloignées.

\section{Proximité et relations aux centres pour l'accès aux services et les pratiques commerciales}

31 Les pratiques citadines liées à deux services de base ont aussi été appréhendées empiriquement : l'éducation et la santé, à travers la prise en compte de la localisation de l'école fréquentée par les enfants du ménage (dans les cas où le ménage en comptait) d'une part, de celle du centre de soins le plus proche utilisé en cas de problème de santé d'autre part. Ainsi, l'accès aux infrastructures scolaires et sanitaires offre un panorama 
contrasté, néanmoins classique, de l'usage de la proximité au regard de la fréquence et des pratiques envisagées. D'un côté, l'accès aux écoles renvoie à une fréquentation privilégiant le contexte local, permise par une offre existante : les enfants sont alors scolarisés majoritairement dans leur quartier pour les premiers cycles. D'un autre côté, l'accès aux soins est plus complexe : il décrit les insuffisances du quartier, mais aussi en contrepartie, l'intégration relative, selon la localisation dans la métropole, aux réseaux urbains et aux ressources présentes dans d'autres zones de la ville. La fréquentation moindre des infrastructures sanitaires, si elle ne traduit pas un ancrage local, peut en revanche éclairer des processus d'acquisition de compétences urbaines à des échelles dépassant largement l'espace du quartier: les centres hospitaliers régulièrement évoqués lors des entretiens E1 peuvent se localiser à des distances de $5 \mathrm{~km}$ de la résidence dans le cas de Tláhuac, $10 \mathrm{~km}$ dans le cas de La Paz, voire jusqu'à $30 \mathrm{~km}$ dans le cas de Milpa Alta.

Deux pratiques commerciales ont également été observées : les courses alimentaires et les courses vestimentaires. La première répond à un approvisionnement quotidien, du moins très fréquent. La seconde renvoie à un poste de dépense important, mais à des déplacements moins courants: d'abord, les emplettes vestimentaires impliquent un type de commerce relativement spécialisé, supposant a priori une zone de chalandise plus large et des points de vente spécifiques plus rares; ensuite, elles renvoient à des circuits commerciaux de produits industriels non périssables, dont la consommation est occasionnelle. Dans nos enquêtes, pour trois quarts des cas, les courses alimentaires se font à proximité du logement: deux fois sur trois dans la colonie de résidence ou dans le quartier voisin, et même, une fois sur dix, dans la même rue que celle de la résidence. La proximité est moins forte, mais tout de même marquée, pour les achats vestimentaires. Les caractéristiques des pratiques commerciales varient selon les quartiers. On observe la fréquentation de marchés hebdomadaires ou plurihebdomadaires dans les rues - les tianguis -, mais aussi de marchés fixes, voire l'articulation avec les chaînes de la grande distribution, surtout dans le cas de Cuautitlán au nord - ces implantations de supermarchés et de centres commerciaux ${ }^{20}$ étant liées aux projets immobiliers d'ensembles de logements sociaux dans cette zone. En dehors de ces lieux situés dans la proximité relative de la résidence, d'autres points en banlieue, dans le péricentre et dans l'hypercentre, participent à construire un système des lieux de consommation large dans l'espace de la ville: entretiens et questionnaires évoquent ainsi le marché populaire et emblématique du centre historique de Tepito, ou encore des centres plus secondaires au niveau métropolitain, mais polarisants localement (San Gregorio pour les habitants de Milpa Alta, grand tianguis du centre de La Paz, centres commerciaux de Nezahualcóyotl, etc.).

$\mathrm{Au}$ total, quatre types d'espaces inégalement pratiqués par les habitants des colonies populaires de Mexico se détachent: le quartier de résidence (appréhendé par le voisinage et la proximité directe), les zones de proximité relative (à l'échelle du quartier et du municipe), les centralités métropolitaines (centre de la ville et centres secondaires), et les autres zones de l'agglomération. Le rapport des habitants des périphéries à leur quartier dépasse donc la seule fonction de logement : il intègre des fonctions économiques et sociales de premier ordre, à lire à différentes échelles, mais il implique aussi la nécessaire prise en compte des réseaux sociaux et politiques dans les pratiques spatiales. 


\section{Les réseaux sociaux dans des contextes de pauvreté et de conflits fonciers} des ressources dans le local. De manière systématique, pour les quatre quartiers étudiés, l'examen au niveau des districts permis par l'enquête EOD met en lumière la plus grande circonscription des pratiques de mobilités quotidiennes destinées à voir un tiers par rapport à d'autres motifs de déplacement (Valette, 2014). Plus finement, le travail empirique (E1 et E2) autorise le suivi des réseaux sociaux actifs des individus (liens familiaux et amicaux) et l'analyse de leur déploiement dans l'espace. Si le réel voisinage - au sens de relations avec les voisins de la résidence - n'est que rarement mis en avant, on a en revanche toujours quelqu'un dans le quartier : dans 8 cas sur 10, un membre de la famille y réside; 7 fois sur 10, le réseau amical considéré comme important réside aussi dans le quartier. Le plus souvent dans l'échantillon étudié ici, ces relations sociales fonctionnent de manière cumulative, la proximité familiale se couplant avec la proximité du réseau amical : 2 enquêtés sur 3 ont, dans leur quartier ou dans le quartier voisin, un membre de leur famille et un ami proche. De plus, réseaux amicaux et familiaux sont fortement imbriqués, du fait des alliances et des réseaux de connaissances tissés à partir du réseau familial. Ainsi, distinguer rigoureusement les deux sphères est très délicat.

L'ancrage des réseaux de sociabilités dans l'espace du quartier soulève plusieurs questions : les ressources que ces réseaux sont susceptibles de fournir aux habitants, les inégalités d'accès à ces ressources qu'ils sous-tendent, l'attachement au quartier, et enfin, l'ambivalence de cette proximité, entre fermeture d'un côté, en termes de liens faibles $^{21}$, et valorisation du capital social de l'autre. La proximité sociale et spatiale facilite les échanges entre les personnes et rend possibles des mécanismes d'entraide indispensables entre les ménages, surtout dans des contextes résidentiels marqués par la pauvreté (Fol, 2010). Les membres de la famille renvoient à des services d'ordre directement pécuniaire (quelle que soit l'échelle spatiale mise en jeu - y compris la transnationale), ou alors, plus inscrits dans l'intimité familiale et/ou domestique: garde d'enfants, aide aux personnes âgées, participation aux activités économiques familiales (gestion d'un commerce par exemple), hébergement, donations foncières, etc. Les réseaux d'amitiés ne sont pourtant pas en reste : ils n'excluent pas l'entraide domestique, l'aide à la mobilité (covoiturage, aide à la motorisation), les prêts d'argent, de logement, de matériel, ou encore l'insertion professionnelle ou le travail collectif (maçonnerie, ouvrages collectifs pour le quartier). Dans un contexte d'emploi souvent non déclaré, donner un coup de main peut être une ressource majeure des habitants enquêtés : pour les uns, c'est un moyen de répartir les tâches; pour les autres, c'est un mécanisme d'insertion dans un marché de l'emploi (même très précaire) qui permet des revenus même minimes (en nature et/ou en argent).

Ces stratégies d'entraide, s'il ne s'agit pas de les décrire avec précision et de manière exhaustive, ni de dessiner les contours d'une improbable sociabilité compensant les difficultés liées au contexte résidentiel, montrent toutefois la fréquence et la force des réseaux sociaux comme ressource localisée dans le quartier. Ce réseau implique néanmoins également des contreparties importantes et des relations de dépendance, à analyser au prisme des histoires familiales dans leur complexité, notamment dans leurs 
dimensions conflictuelles, marquantes dans la structuration des stratégies de sociabilités.

Les sociabilités qui déterminent lourdement l'accès aux ressources locales s'inscrivent dans la sphère domestique, mais aussi dans la sphère publique. Dans des contextes résidentiels où l'illégalité de la propriété et le non-respect des normes urbanistiques sont dominants, et où les infrastructures urbaines de base (eau, électricité, assainissement, voirie) sont déficientes, voire absentes, la scène, sociale et politique, renvoie dans ces quartiers aux enjeux des organisations populaires et des actions collectives autour de la régularisation physique et foncière. Cette régularisation est le fruit de la négociation permanente et sur le long terme entre résidents et autorités, permettant la consolidation, la titrisation et l'urbanisation progressive. En effet, c'est bien l'articulation de la dimension foncière, spécifique au Mexique, avec celle de l'organisation sociale - elle-même dépendant des rapports de force instaurés par les statuts fonciers du point de vue de la légitimité de l'occupation du sol - qui permet la lecture fine des ressources urbaines (notamment pour l'accès au logement) dans le contexte résidentiel local. Les articulations des trois pans de la régularisation (équipement, propriété et usage) décrivent des trajectoires plurielles des quartiers, des ménages et de la dimension évolutive de l'existence des ressources dans le local (Valette, 2014). Le poids du foncier et celui de l'organisation politique sont donc déterminants dans le développement et l'efficacité du réseau social comme ressource intrinsèque, mais aussi comme vecteurs d'autres ressources, comme l'accès aux services urbains ou à la sécurité foncière (en propriété ou en location). En cela, l'organisation socio-politique est au cœur des processus de maturation et de production de l'espace ordinaire pour des espaces souvent analysés comme marginaux (Lombard, 2014, 2015).

$\mathrm{Au}$ total, l'espace public des colonies populaires périphériques appréhendé au travers des sociabilités, même s'il n'est pas délimité avec précision, semble toutefois rassembler les deux dimensions essentielles à la production de relations hétérogènes dans ces contextes résidentiels : rencontre occasionnelle avec l'étranger et rencontre quotidienne avec le connu (Duhau et Giglia, 2004, p. 186). Cette double expérience urbaine participe du sentiment d'ancrage dans le quartier: les relations de proximité sont plurielles, contribuant à un sentiment d'appartenance locale, couplé d'une perception de connexion au reste de la ville, loin de la seule position marginale. Néanmoins, dans un contexte de précarité économique et foncière, comme sous d'autres latitudes, le lien fort au local, politisé, conflictuel parfois, peut aussi renvoyer à un resserrement de l'espace social des habitants, et à une relative exclusion des autres réseaux urbains.

39 À travers le suivi des pratiques, l'ancrage en quartier populaire périphérique permet $a$ priori la fixation d'une partie importante du réseau social dans la proximité résidentielle, mais n'exclut pas (au contraire) la fréquentation de lieux plus éloignés dans la ville. Les stratégies des habitants, dans les configurations de leurs ancrages au quotidien, renvoient à la manière dont sont mobilisées les ressources de la proximité certes, mais ces stratégies dépendent aussi des caractéristiques du contexte local en luimême. En particulier, une relative fonctionnalisation permet une diversification des ressources sur place. 


\section{La fonctionnalisation économique du quartier : les ressources du local}

40

Observer les ressources potentielles du contexte résidentiel lui-même passe par l'exploration des différentes fonctions économiques et sociales présentes à plusieurs échelles dans le quartier d'une part, des transformations qui ont permis ce caractère fonctionnel d'autre part.

\section{Le développement des commerces et des services à l'échelle du quartier}

41 Malgré la poursuite de la domination de la Ciudad de México en termes d'emplois ${ }^{22}$, la « pénétration progressive de l'emploi dans les périphéries » (Paquette, 2010, p. 168) est encore difficilement lisible du point de vue des recensements économiques : en 2009 au moment des enquêtes, les quatre municipalités périphériques ici étudiées concentrent 4,5\% (cumulés) des unités économiques de la ZMVM, 2,1\% des emplois métropolitains, mais seulement $1,2 \%$ de la valeur de la production (pour $4,4 \%$ de la population totale) (INEGI, 2012). Toutefois, ce poids économique faible dans la balance de la métropole ne doit pas masquer le développement de fonctions autres que résidentielles.

L'ensemble des activités économiques qui sont susceptibles d'animer les quartiers étudiés peut être exploré de manière systématique par le répertoire statistique national des unités économiques (DENUE) qui recense les établissements au niveau des îlots ${ }^{23}$. En combinant ces résultats à nos relevés de terrain, il est possible d'approcher la couverture des quartiers par un tissu commercial et un panel de services (illustrations 7 et 8). Alors qu'en moyenne dans la métropole, on compte une unité économique pour 26 habitants, dont un commerce pour environ 50 habitants (une unité pour 8 et un commerce pour 20 dans la délégation centrale de Cuauhtémoc), ce ratio est, sans surprise, moindre dans les terrains d'étude, mais différencié selon la zone: San Pablo à Milpa Alta semble globalement mieux couverte par ce réseau économique (1 unité pour 26 habitants, 1 commerce pour 40 ), tandis que les densités sont plus faibles à Cuautitlán ( 1 commerce pour 67 habitants) et dans les quartiers à l'est de La Paz ( 1 unité pour 40 habitants en moyenne, dont un commerce pour 60 et une unité d'activités de service pour 160). Ces fonctions sont donc loin d'être absentes. 
Illustration 7 - Les fonctions commerciales dans quatre quartiers populaires périphériques

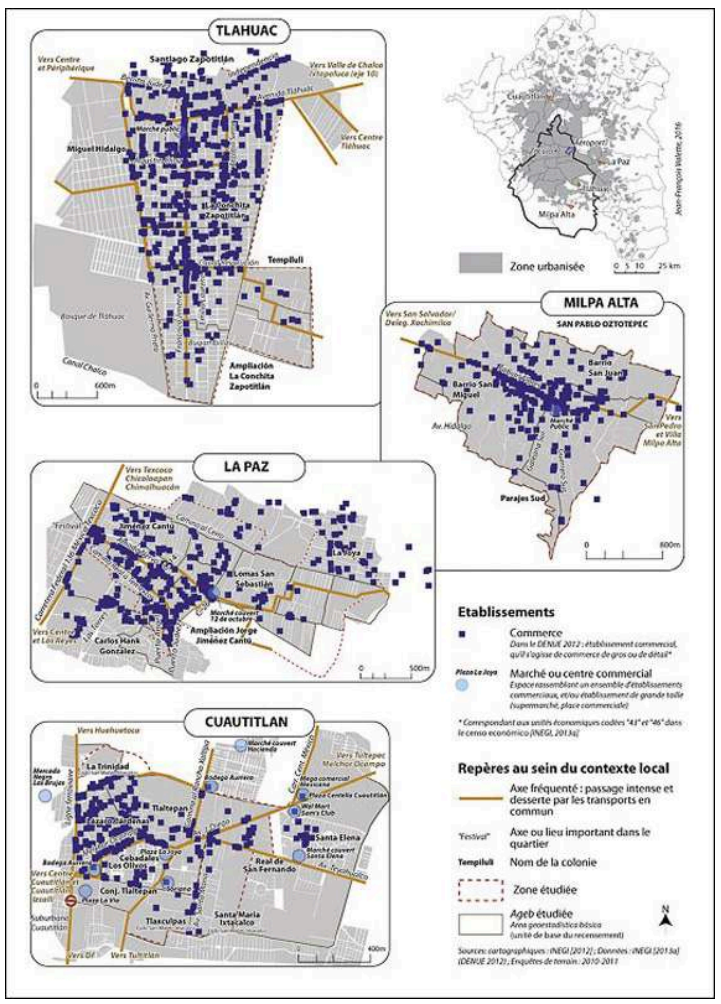

Illustration 8 - Les fonctions de service dans quatre quartiers populaires périphériques

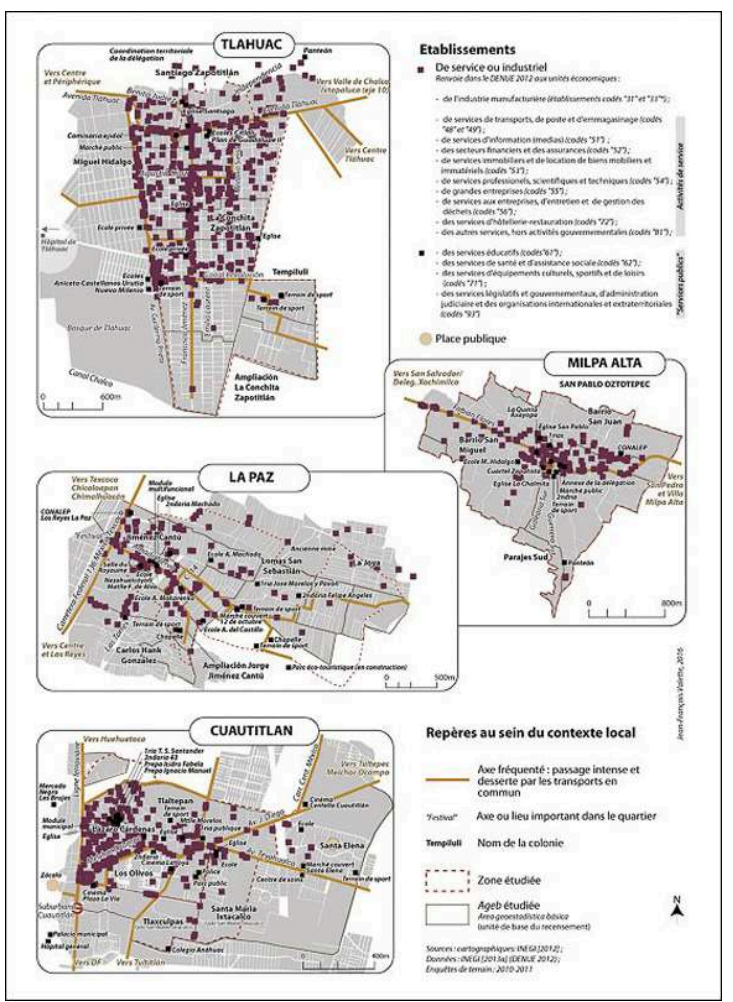

43 On observe ainsi d'abord un réseau dense de petites entreprises (micro-commerces, ateliers, etc.), qui permet de lire la diversité du tissu socio-économique des quartiers 
populaires périphériques (illustration 7). Les concentrations spatiales s'observent le long des axes principaux de circulation et dans les zones de centralité et de souscentralité. Mais si des lieux de réelle centralité commerciale et de polarisation de la consommation existent bel et bien, il faut aussi savoir compter avec une dimension intermittente et plus complexe de la vie des unités économiques dans ces espaces ${ }^{24}$. Par ailleurs, en plus de fournir une partie des emplois et de l'activité économique nécessaire à la vie urbaine, le quartier peut aussi accueillir une partie des services publics de proximité essentiels (illustration 8). Par exemple, à l'est de La Paz, dans un quartier comptant 13000 habitants, on recense près de 400 commerces, services et industries et près d'une trentaine de services publics. L'implantation des groupes scolaires - aux statuts variés (privé, public) - renvoie à une couverture de proximité dans la plupart des quartiers et participe aussi des centralités locales. Ces espaces, marqués par la pauvreté ne sont donc pourtant pas des déserts fonctionnels.

\section{La rue et la sphère domestique comme espaces fonctionnels}

Vivre dans son quartier, y entretenir des sociabilités et s'y attacher induisent la présence relative de lieux de citadinité, autrement dit d'un espace public au sein duquel la scène locale prend place. Par exemple, la présence de stands commerciaux divers et de places publiques où les échanges et les rencontres sont possibles donne au contexte résidentiel une résonnance importante en termes de sociabilités (Duhau et Giglia, 2004, 2008). Ce rôle de la rue - et de l'espace public en général - est encore renforcé lorsqu'existent des aménagements collectifs plus vastes où la circulation automobile est proscrite. C'est notamment le cas dans les colonies Jiménez Cantú à La Paz et Lázaro Cárdenas à Cuautitlán: au cœur de ces deux colonies - les plus anciennement urbanisées des fronts étudiés ici -, la présence d'un lieu public autour d'une annexe des services municipaux autorise les échanges entre habitants (illustration 8). Ainsi, le modulo multifuncional ou municipal dans ces deux colonies est le lieu de contacts entre les résidents et les services municipaux (relais de l'administration, cadastre, État civil, police), mais aussi le lieu de discussions, de vente occasionnelle, voire de réunions et de festivités (illustrations 9 et 10). 
Illustrations 9 et 10 - Module multifonctionnel à La Paz : espace public et sociabilités

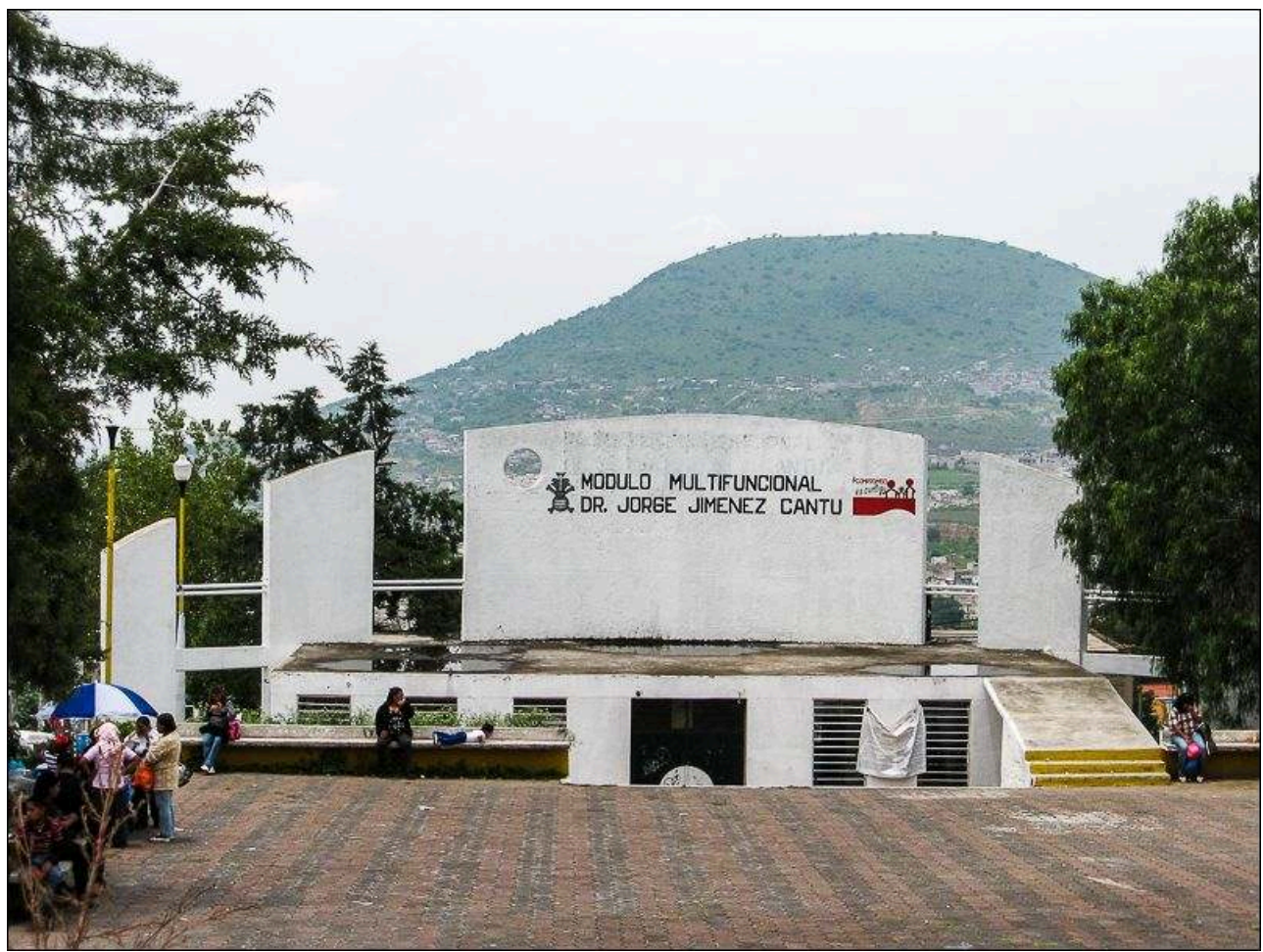

Place aménagée avec circulation interdite. Bâtiment abritant une annexe municipale et une antenne de la police locale.

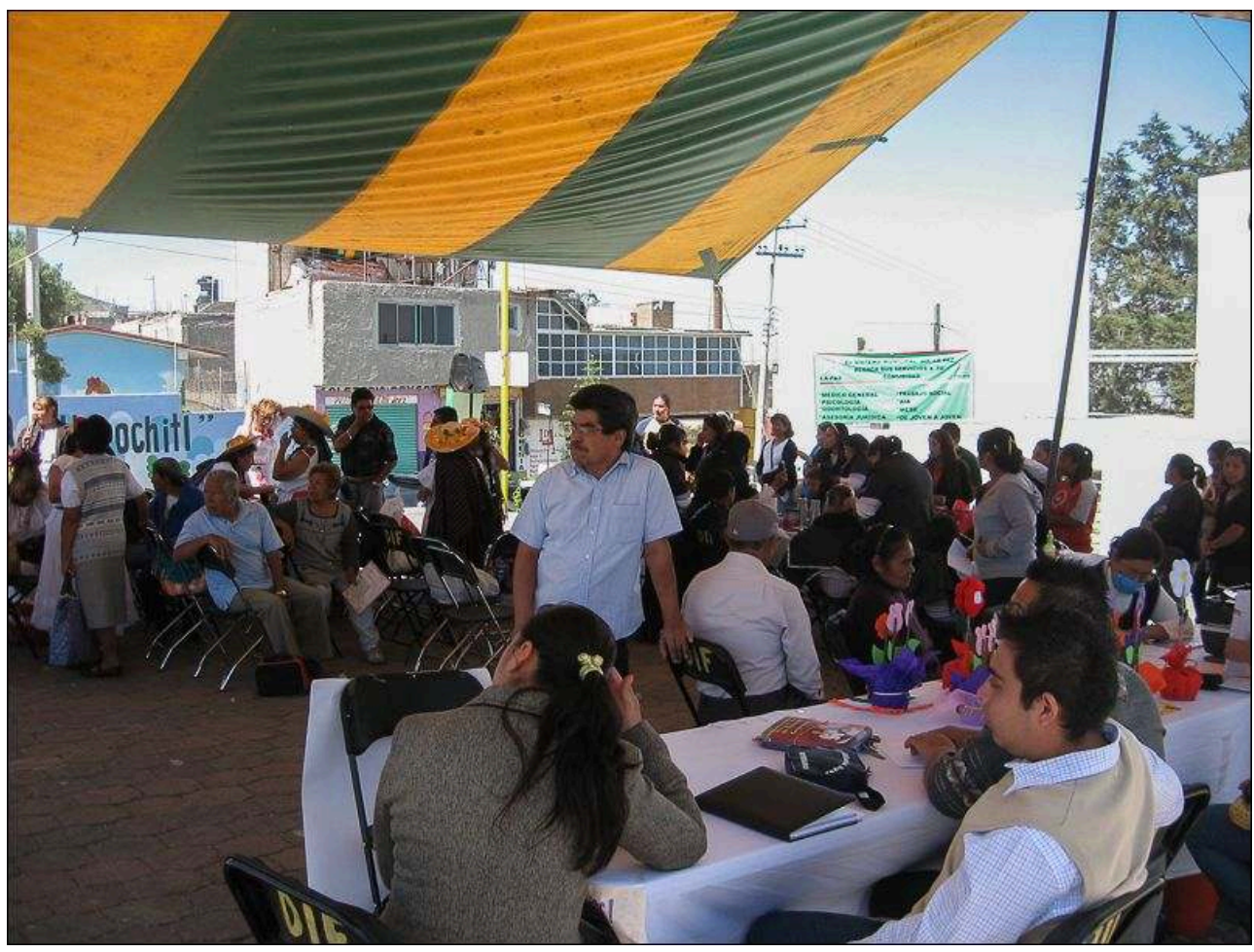

Réunion de quartier organisée par les services sociaux du DIF (Développement intégral de la famille). Auteur : J.-F. Valette, 2010. 
45 À l'échelle de la rue, dans les quatre quartiers, le suivi d'axes particulièrement structurants met en lumière premièrement, la mixité des activités économiques dans la proximité résidentielle directe, deuxièmement, la pénétration de cette fonctionnalisation parfois loin en périphérie et troisièmement, la force du gradient de consolidation du peuplement qui structure ce tissu d'activités. La densité des activités est liée à celles des constructions (et à la durcification de ces dernières), mais aussi à la présence dans le contexte direct des services de base (électricité, eau, voirie) et plus largement, à l'importance du passage sur la voie publique - elle-même liée aux dimensions précédentes.

Enfin, le rapport au quartier rendu possible par la fonctionnalisation économique du lieu est lié au développement de l'auto-entrepreneuriat. En effet, l'articulation nécessaire entre logement et emploi est à comprendre par rapport à l'imbrication des réseaux sociaux, familiaux et économiques, au cœur des mécanismes d'ancrage local dans les colonies populaires périphériques de Mexico ${ }^{25}$. L'éventuelle fonctionnalisation économique de l'espace domestique ne doit néanmoins pas être confondue avec la fonctionnalisation dans son ensemble: la présence de l'activité dans le logement ne concerne pas toutes les activités économiques évoquées d'une part (entre 10 et $30 \%$ des enquêtés de notre échantillon); une partie des commerces, ateliers et services ne renvoie pas à la fonctionnalisation d'une construction initialement prévue pour le logement, mais à des locaux spécifiques loués par des non-résidents du quartier d'autre part. L'usage de la résidence pour l'auto-entrepreneuriat, s'il traduit les stratégies sociales et économiques des ménages, renvoie bien aux marqueurs d'un ancrage dans les colonies populaires. Il s'agit d'un des signes de la production progressive de l'espace urbain, qui implique une manière d'habiter spécifique aux quartiers populaires périphériques, basée sur la rentabilisation et la valorisation sur le long terme de la résidence (Hiernaux et Lindón, 2003 ; Giglia, 2012).

La fonctionnalisation du quartier témoigne des différentes temporalités de l'investissement de l'espace local. Mais elle invite aussi à appréhender la variété des ancrages, à plusieurs échelles, et les inégalités d'accès à la ville qu'ils sous-tendent.

\section{Du rapport au quartier à l'ancrage urbain : point de vue des individus}

$48 \mathrm{Au}$ niveau des individus, replacer les pratiques et les ressources du quartier dans le contexte de la ville dans son ensemble et au-delà permet de mieux analyser les systèmes de lieux métropolitains mobilisés par les habitants, et les liens entre le quartier de résidence et les autres lieux de leur espace de vie. Quels bassins de vie sont dessinés par ces pratiques multiples dans la ville? Quels ancrages se construisent par ces circulations?

\section{Le quartier « ne suffit pas »}

Les processus de citadinisation et de développement des compétences urbaines dans la recherche des ressources sociales et économiques impliquent l'ancrage local autant que la pratique de la ville (Berry-Chickhaoui et Deboulet, 2000 ; Baby-Collin, 2005 ; Florin et al., 2007 ; Bouillon, 2009 ; Massot, 2010 ; Berroir et al., 2016). Sortir de son quartier très 
régulièrement constitue une dimension majeure de la citadinité et de l'ancrage urbain. Même si les quartiers étudiés ne renvoient pas à des lieux de l'anomie et de l'absence de centralité et d'urbanité en général, il ne s'agit pas non plus de nier les réelles carences et la perception forte d'insuffisance du contexte local. La pratique de la ville, en dehors du quartier, est à la fois une condition systématique pour disposer de ressources (s'approvisionner), en même temps qu'un marqueur de différenciation sociale important - l'échelle métropolitaine étant un territoire de référence plus difficile à cerner pour comprendre la conquête de la ville (Baby-Collin, 2005, p. 155 ; Lazo, 2012, p. 25).

50 La nécessité du lien à la ville, voire à d'autres lieux extra-métropolitains, vient ici du manque de ressources ressenti dans le local - quelle que soit la maille utilisée - et de la perspective de devoir trouver ces ressources ailleurs. C'est par exemple le cas des disponibilités foncières. Cette dimension est importante dans la projection de l'installation des nouvelles générations, certains jeunes ménages souhaitant accéder à la propriété ou à des locations moins onéreuses d'une part, d'autres, déjà propriétaires, souhaitant élargir leur patrimoine - souvent pour les descendants - d'autre part. À travers cette ressource urbaine, trois dimensions ressortent. Premièrement, la dépendance des contextes résidentiels étudiés par rapport aux autres espaces de la ville paraît encore forte. Deuxièmement, l'intégration de ces zones au travers d'une banalisation par densification et consolidation est en marche. Troisième point enfin, le dynamisme conséquent de cette intégration - impliquant la nécessaire ouverture du quartier sur d'autres espaces urbanisés ou à urbaniser - est visible. Entre extension et saturation, les quartiers populaires périphériques ne sont pas des lieux d'ancrage isolés, mais bel et bien les espaces d'ancrages pluriels autour desquels se développent des bassins de vie (au moins) métropolitains.

51 Enfin, l'utilisation du quartier populaire par des populations extérieures - entendues comme celles qui n'y résident pas -, du fait des fonctions urbaines (emplois, services) s'y localisant, permet de souligner le caractère allochtone de la pratique du quartier. Cette dimension constitue aussi un marqueur important de l'insertion dans les réseaux sociaux et économiques de la ville : elle tend à hisser ces espaces au rang de quartiers banals, où vont et viennent résidents et autres urbains.

\section{Des bassins de vie différenciés en formation?}

52 Le suivi de quelques parcours appréhendés empiriquement illustre et rend compte de différentes configurations typiques d'ancrages. Des associations entre les activités des habitants permettent de lire des degrés d'usage du local, qui eux-mêmes participent à l'identification de types de rapport au quartier; ces rapports au quartier permettent quant à eux la construction d'une typologie exploratoire des rapports résidentiels différenciés, combinant usages du logement, du quartier et de la ville (Authier, 2001). Si les quelques types de systèmes de lieux présentés ici n'ont pas vocation à la représentativité, l'exhaustivité, ou l'exclusivité des configurations possibles, ils peuvent toutefois nourrir la réflexion sur les combinaisons complexes des circulations à Mexico, dans et à partir des colonies populaires, et sur les inégalités socio-spatiales que ces combinaisons permettent de mettre au jour.

53 Les profils emblématiques d'ancrages mobilisent différents registres, à la fois relatifs à la spatialisation des pratiques quotidiennes, à celle des systèmes résidentiels, mais 
aussi aux représentations associées à la position dans le quartier (Valette, 2014). Pour cerner l'ancrage dans sa complexité, la pratique doit aussi être analysée par rapport à un grand ensemble de variables appréhendées au cours des entretiens E1 : le parcours résidentiel, la participation à la vie collective du quartier, spécifique des fronts pionniers urbains, le sentiment d'appartenance au lieu, la relative connaissance de la proximité résidentielle élargie (quartier et alentours), et la perception de l'insécurité dans le local, dans un contexte violent très médiatisé et de méfiance généralisée ${ }^{26}$. Au total, les modalités de ces dimensions se croisent pour se décliner en plusieurs profils d'ancrages non exclusifs, plus ou moins marqués par i) la polarisation dans l'espace local, ii) le rôle de pivot du local dans une circulation à plusieurs échelles, iii) la multipolarisation des pratiques et/ou iv) le caractère temporaire ou faible de l'ancrage.

D'abord se distinguent les profils d'ancrage où le système de lieux mis en relation s'inscrit de manière privilégiée dans le contexte local. Ces types d'ancrages sont qualifiables d'autochtones : ici, c'est la force du localisme de la trajectoire résidentielle, le relatif monopole du quartier dans le système de mobilités et l'investissement politique et affectif qui sont soulignés, mais ces configurations n'impliquent pas forcément la captivité. L'exemple de Fernando, à Ampliación La Conchita à Tláhuac, 73 ans en 2010, peut illustrer ce type (illustration 11-1). En plus d'un parcours résidentiel fortement inscrit dans le contexte local (enfant d'ejidatarios du village de Zapotitlán), ses pratiques quotidiennes sont très centrées sur la proximité résidentielle : l'école de ses petits-enfants se situe dans la colonie voisine, son activité principale (maraîchage) est localisée à Ampliación, sa deuxième résidence, ainsi que celle d'une partie de ses enfants, sont situées dans la colonie voisine de La Conchita, tandis que son réseau amical et ses connaissances liées à la communauté de l'ejido sont localisés dans le pueblo. La configuration de Fernando n'implique pas pour autant l'exclusivité de la situation des lieux de son espace de vie dans ce seul contexte local : il garde aussi une partie de ses repères dans le centre-ville de Mexico, où il a travaillé auparavant.

L'ancrage polarisé dans le local peut aussi renvoyer à des situations de bassins de vie métropolitains resserrés, par adoption : on fait ici référence à une forme de pratiques spatiales concernant à la fois des profils de nouveaux arrivants (nouvelle génération), comme d'ancrés (deuxième génération). Pour ces populations, souvent jeunes, mais aussi féminines dans nos enquêtes, la force du local peut renvoyer à un attachement au quartier et/ou à un relatif enfermement faute de mieux - n'excluant cependant pas des articulations avec le reste de la ville, voire au-delà.

Un deuxième grand type d'ancrage témoigne de pratiques quotidiennes mixtes, c'est à dire localisées à la fois dans la proximité résidentielle et au-delà. Dans ce type de configurations, la résidence joue un rôle de pivot autour duquel se structurent une mobilité citadine développée, ainsi qu'un ancrage politique et social. Le champ spatial de ces mobilités peut se situer sur plusieurs échelles ou être relativement limité dans la métropole. L'articulation du système d'activités sur plusieurs échelles peut être illustrée avec l'exemple de Carmen, 21 ans en 2010, résidente à Tempiluli depuis 2005 (illustration 11-2) : la localisation de ses activités dans le contexte hyper-local se couple d'une fréquentation régulière d'autres lieux, en dehors de la proximité directe. Ses pratiques estudiantines et sociales (réseau amical et loisirs) se répartissent entre Xochimilco (fréquenté plusieurs fois par semaine) et Iztapalapa (fréquenté quotidiennement), mais c'est tout de même autour du pôle de la résidence principale 
parentale que se structure ce système de lieux. Malgré le caractère complémentaire des autres lieux dans sa recherche des ressources urbaines, le poids du système résidentiel familial dans la configuration de ses circulations dans la métropole reste important.

D'autres ancrages citadins peuvent s'inscrire plus largement dans l'espace métropolitain. L'ensemble des pôles pratiqués de manière intense dans le reste de la ville témoigne du rôle de point de départ des ancrages locaux dans le quartier résidentiel pour les «explorations extérieures » des habitants - évoquées par J. Rémy (1996). L'ancrage citadin multipolarisé articule ainsi le poids d'un contexte local investi socialement, et des ancrages secondaires dans d'autres parties de la ville. Ces autres lieux importants de l'espace des activités - au sein d'un espace de vie plus complexe peuvent impliquer un bassin de vie large, à l'échelle de la métropole. L'exemple de Juán est en cela caractéristique (illustration 11-3): alors que son parcours résidentiel renvoie au modèle de Turner (province-centre-périphérie), Juán pratique régulièrement d'autres espaces de la métropole que son lieu de résidence. En plus de ses liens familiaux localisés dans l'État de Oaxaca - qu'il fréquente deux fois par an -, il se rend tous les mois chez ses beaux-parents à Naucalpan, de l'autre côté de la ville, mais aussi chez sa fille, qui réside dans le quartier central de la Villa Guadalupe - où il a lui-même vécu pendant 18 ans -, et une fois par semaine dans une église du DF, où il a tissé un solide réseau amical. Juán n’a pas pour autant un ancrage local faible: au contraire, c'est dans ce contexte local qu'il indique faire ses courses, avoir amené ses enfants à l'école, gérer son petit commerce et avoir un solide réseau d'amis; il signale se sentir profondément de son quartier, qu'il a mis vingt ans à apprivoiser, le travail de régularisation foncière et de consolidation y a été laborieux et coûteux. Ici, la mobilité urbaine à l'échelle de la ville n'exclut en rien la pratique intense ou le rôle central du quartier de résidence. Pour reprendre les termes de J.-Y. Authier, «l'ancrage dans le quartier et la mobilité urbaine, loin de constituer deux modes opposés d'habiter, fonctionnent de pair » (2001, p. 166).

Enfin, certains profils mettent en lumière un ancrage de prime abord faible et où les ajustements du système de mobilité semblent temporaires. Peu visibles sur la scène locale, car a priori peu investis ni attachés au lieu, ces urbains peuvent avoir des pratiques très diverses, n'impliquant pas uniquement une faiblesse des usages locaux, quotidiens ou résidentiels, et pouvant renvoyer sous certains aspects aux types d'ancrages précédemment présentés. Ces circulations témoignent, en dépit du faible attachement et investissement local, du rôle pivot que peut jouer la résidence dans ces quartiers, y compris pour ceux qui y connaissent une position socio-résidentielle précaire et qui ne s'y projettent pas. L'exemple de Luis à Lomas San Sebastián peut fournir quelques clefs de lecture de cette catégorie difficile à cerner (illustration 11-4) : sa résidence à $\mathrm{La}$ Paz constitue un nouveau sacrifice relativement douloureux dans le sens où il a quitté son village - dans l'État de Puebla, où vivent sa femme et ses trois enfants -, pour rechercher un emploi dans la métropole, comme il l'avait déjà fait une première fois quelques années auparavant. Hébergé par sa sœur dans des conditions difficiles - sans électricité en $2010^{-}$, il travaille comme ouvrier dans une usine d'Iztapalapa, mais localise l'essentiel de ses autres activités au sein de la colonie de résidence actuelle, notamment à travers les actions politiques du groupe de voisins condition requise pour accéder à un meilleur équipement en services urbains. Toutefois, le lieu qu'il érige comme central et qu'il espère rejoindre au plus vite reste son village, considérant son étape actuelle comme transitoire. 
$\mathrm{Au}$ total, l'effort typologique n'a de portée qu'exploratoire, dans le sens où il est l'occasion de questionner les variables à intégrer à l'analyse des ancrages dans ces quartiers populaires, tout comme le poids et les portées différenciées de ces variables. Systèmes résidentiels, pratiques quotidiennes, activités diverses liées à la recherche de ressources urbaines, réseaux familiaux et sociaux, mais aussi investissements politiques, représentations de la position résidentielle et projets résidentiels fournissent autant d'éléments renseignant l'ancrage des habitants, la structuration de leur système de mobilités et les différenciations socio-spatiales lourdes en termes d'accès à la ville.

Illustration 11 - Profils d'ancrages
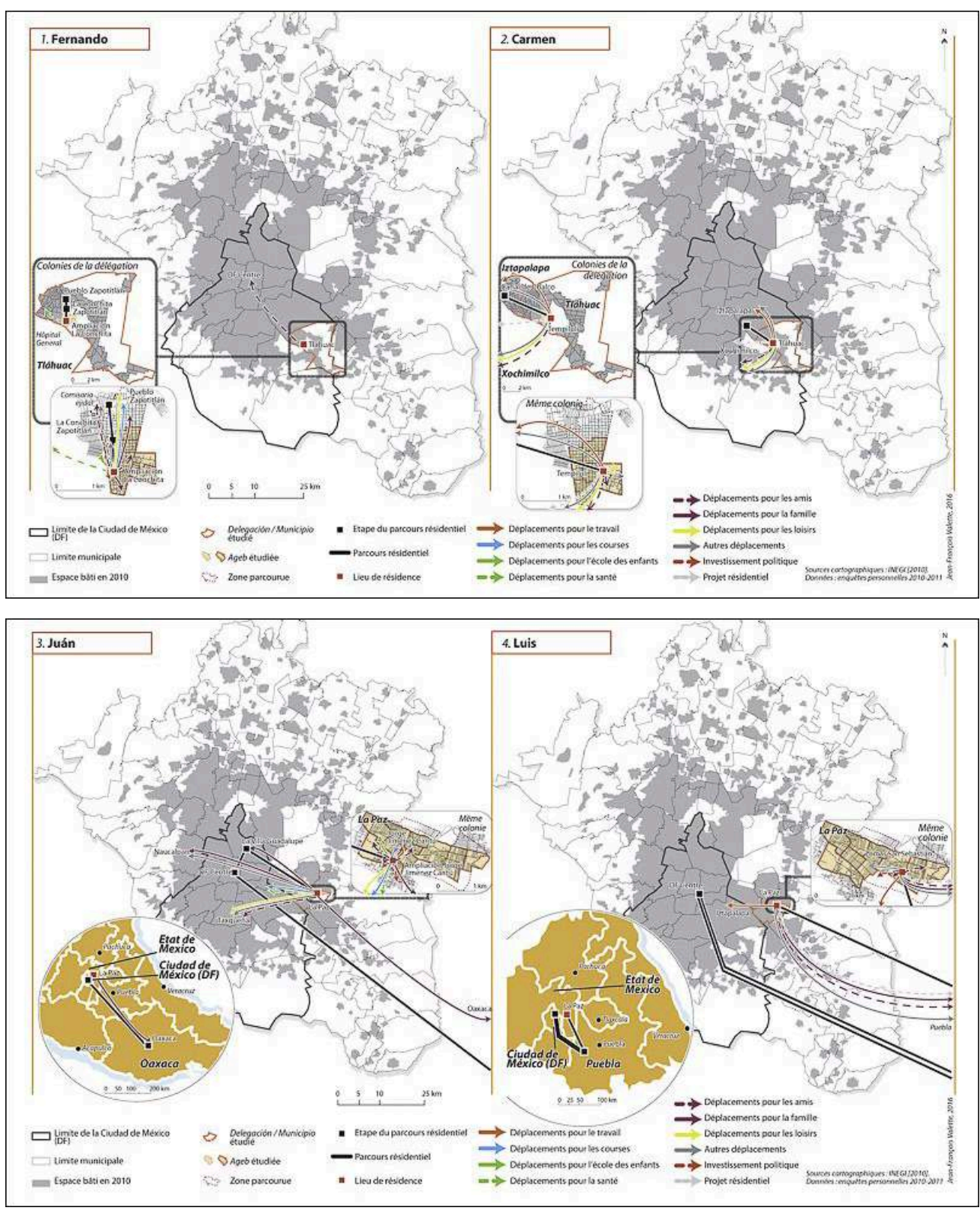


\section{Conclusion}

S'intéresser aux pratiques citadines et aux ressources dans le lieu et $d u$ lieu fournit des informations précieuses sur la manière dont les habitants investissent leur espace local, se l'approprient et se sentent progressivement y appartenir, alors même que ces dimensions ne vont pas de soi dans ces contextes résidentiels de fronts pionniers urbains initialement irréguliers. Les pratiques de mobilités quotidiennes renvoient ainsi à deux caractéristiques majeures. D'une part, une relative intensité des déplacements dans la ville est soulignée: le quartier n'apparaît pas tant comme l'espace de la sédentarité, ni souhaitée ni subie ; au contraire, il s'intègre relativement au reste de la métropole. D'autre part, l'ancrage territorial renvoie à la manière dont sont mobilisées les ressources sociales et économiques dans la proximité.

Deux dimensions de l'ancrage quotidien sont alors soulignées. Premièrement, la maturation de ressources urbaines dans le local est mise en lumière: l'activité économique et la présence de services ont une importance fondamentale dans la pratique et la représentation du quartier, et dans les dynamiques de fonctionnalisation participant aux ancrages locaux. Deuxièmement, l'inscription de ce relatif localisme dans des systèmes de lieux pluriels ressort nettement: des bassins de vie sont mis en évidence autour de la résidence en quartier populaire et une lecture de l'intégration au reste de la ville est possible.

Si ces entrées et ces résultats relatifs aux liens à la ville de ces espaces en termes de ressources urbaines restent classiques, les pratiques quotidiennes révèlent des formes de sociabilités particulières, à comprendre notamment autour de l'organisation du système résidentiel, de la consolidation physique et de la dimension foncière des contextes étudiés. Quatre grands types non exclusifs d'ancrages au quotidien peuvent ainsi être approchés : polarisés par le local, structurés à plusieurs échelles à partir du local, multipolarisés dans la métropole voire au-delà, et faibles ou temporaires. En cela, les différents modes d'ancrages résidentiels dans l'espace politico-juridique sont une grille de lecture essentielle pour analyser les ressources urbaines des périphéries de Mexico et les inégalités socio-spatiales qu'elles sous-tendent.

\section{BIBLIOGRAPHY}

Authier J.-Y. (dir.), 2001. Du domicile à la ville. Vivre en quartiers anciens. Paris, Anthropos, coll. Villes, $214 \mathrm{p}$.

Avellaneda P., Lazo A., 2011. Aproximación a la movilidad cotidiana en la periferia pobre de dos ciudades latinoamericanas. Los casos de Lima y Santiago de Chile. Revista Transporte y Territorio, $\mathrm{n}^{\circ}$ 4, Universidad de Buenos Aires, p. 47-58, http://www.rtt.filo.uba.ar/RTT00404047.pdf

Baby-Collin V., 2005. Des marges dans la ville : mobilités citadines et métissage de l'urbanité. In Capron G., Cortes G., Guetat-Bernard H. (dir.), Liens et lieux de la mobilité. Ces autres territoires. Belin, Coll. Mappemonde, p. 145-165. 
Bacqué M.-H., Fol S., 2007. L'inégalité face à la mobilité : du constat à l'injonction. Swiss Journal of Sociology, $\mathrm{n}^{\circ}$ 33(1), p. 89-104

Berger M., Aragau C., Rougé L., 2014. Vers une maturité des territoires périurbains ? EchoGéo, $\mathrm{n}^{\circ} 27$, http://echogeo.revues.org/13683 ; DOI : 10.4000/echogeo.13683

Berroir S., Delage M., Fleury A., 2016. Le commerce dans la fabrique périurbaine : centralités commerciales et pratiques habitantes au nord de la métropole parisienne. Géographie, économie, société, $\mathrm{n}^{\circ} 1$, vol. 18 , p. 35-57.

Berry-Chikhaoui I., Deboulet A. (dir.), 2000. Les compétences des citadins dans le Monde arabe. Penser, faire et transformer la ville. IRMC Khartala-URBAMA, $402 \mathrm{p}$.

Bertrand M., 2010a. De l'accès au logement à la relation domicile-travail : enjeux sociaux et spatiaux des mobilités dans la région du Grand Accra, Ghana. Revue Tiers Monde, n²01/1, A. Colin, p. 87-106.

Bertrand M., 2010b. Migration internationale et métropolisation en Afrique de l'Ouest : le cas des Zabrama du Grand Accra, Ghana. Espace, Populations, Sociétés, n 2-3, p. 307-320.

Bertrand M., 2011. De Bamako à Accra. Mobilités urbaines et ancrages locaux en Afrique de l'Ouest. Karthala, Paris, 376 p.

Bouillon F., 2009. Les mondes du squat. PUF, Le Monde, 245 p.

Brevet N., 2008. Mobilités et processus d'ancrage en ville nouvelle : Marne-la-Vallée, un bassin de vie ? Étude des mobilités résidentielles et des mobilités quotidiennes. Thèse d'urbanisme, IUP, Université Paris-Est, 546 p.

Brunet R., Ferras R., Théry H., 1992. Les mots de la géographie. Dictionnaire critique, La Documentation française, Reclus, Montpellier-Paris, $470 \mathrm{p}$.

Carpentier S., Gerber P., Klein O., Schneider M., 2010. Du bassin de vie aux espaces de vie. Une approche multiscalaire des mobilités locales. Groupe Dupont, Colloque Geopoint « Les échelles pour les géographes et les autres. Cultures, finalités et pratiques scalaires », $18^{\text {ème }}$ Biennale de Géographie, Université d'Avignon, 3-4 juin 2010.

Contreras Y., 2012. Cambios socio-espaciales en el centro de Santiago de Chile: Formas de anclarse $y$ prácticas urbanas de los nuevos habitantes. Thèse de géographie, Université de Poitiers, Pontifica Universidad Católica de Chile, 403 p.

Di Meo G., 1994. Épistémologie des approches géographiques et socio-anthropologiques du quartier urbain. Annales de Géographie, A. Colin, n 577, p. 255-275.

Dubucs H., Giroud M., 2014. Systèmes d'activités et pratiques urbaines dans la circulation : continuités ou complémentarités? In Imbert C., Dubucs H., Dureau F., Giroud M., D'une métropole à l'autre. Pratiques urbaines et circulations dans l'espace européen. A. Colin, Recherches, Paris, p. 363-408.

Duhau E., 2003. División Social del espacio metropolitano y movilidad residencial. Papeles de Población, n³6, UAEM, p. 161-210.

Duhau E., Giglia Á., 2004. Espacio público y nuevas centralidades. Dimensión local y urbanidad en las colonias populares de la ciudad de México. Papeles de Población, $n^{\circ}$ 41, UAEM, Toluca, México, p. $167-194$

Duhau E., Giglia Á., 2007. Nuevas centralidades et prácticas de consumo en la Ciudad de México: del microcomercio al hipermercado. EURE, vol. $33, n^{\circ} 98$, p. 77-95. 
Duhau E., Giglia Á., 2008. Las reglas del desorden: habitar la metrópoli. UAM-A, México, 570 p.

Dureau F., 2002. Les systèmes résidentiels : concepts et applications. In Lévy J.-P. et Dureau F. (dir.), L'accès à la ville. Les mobilités spatiales en question. Paris, L'Harmattan, coll. Habitat et sociétés, p. 355-382.

Dureau F., Beauchemin C., Coubes M.-L., Delaunay D., 2006. Les mobilités spatiales dans des contextes en évolution : analyse croisée des deux dynamiques. In Antoine P. et Lelièvre E. (dir.), États flous et trajectoires complexes. Observation, modélisation, interprétation. GRAB, coll. Méthodes et savoirs, INED, CRD, p.157-194.

Dureau F., Giroud M., Lévy J.-P., 2014. L'observation des mobilités quotidiennes. In Imbert C., Dubucs H., Dureau F., Giroud M., D'une métropole à l'autre. Pratiques urbaines et circulations dans l'espace européen. A. Colin, Recherches, Paris, p. 81-132.

Dureau F., Gouëset V., 2010. Formes de peuplement et inégalités de déplacements. L'évolution des mobilités quotidiennes dans deux périphéries populaires de Bogota : Soacha et Madrid, 1993-2009. Revue Tiers Monde, n² 201/1, A. Colin, p. 131-156.

Dureau F., Gouëset V., Le Roux G., Lulle T., 2014. L'inégalité d'accès aux ressources urbaines dans des quartiers périphériques en mutation nouvellement desservis par le Transmilenio à Bogotá (Colombie). Cahiers de géographie du Québec, vol. 58, n 163, p. 7-38.

Dureau F., Lulle T., Souchaud S., Contreras Y., 2014. Mobilités et changement urbain. Bogotá, Santiago et São Paulo. PUR, Coll. Espaces et Territoires, 440 p.

Florin B., Baby-Collin V., Bouillon F., Didier S., Dorrier-Apprill E., 2007. Bouger, s'ancrer. In Dorrier-Apprill E., Gervais-Lambony P., Vies citadines. Belin, Coll. Mappemonde, Paris, p. 81-108.

Florin B., Semmoud N., 2010. Mobilités résidentielles et territorialisations dans les villes du Maghreb : entre exclusion et intégration. Espace, Populations, Sociétés, n² 2-3, p. 365-377.

Fol S., 2009. La mobilité des pauvres. Belin, Coll. Mappemonde, 262 p.

Fol S., 2010. Mobilité et ancrage dans les quartiers pauvres : les ressources de la proximité. Regards Sociologiques, $n^{\circ} 40$, p. 27-43.

Giglia Á., 2012. Habiter la précarité et vivre ensemble dans la ville informelle. In Frelat-Kahn B., Lazzarotti O. (dir.), Habiter. Vers un nouveau concept ? A. Colin, Coll. Recherches, Paris, p. 229-244.

Gouëset V., Dureau F., 2006. Introduction à l'Amérique latine des villes. In Dureau F., Gouëset V., Mesclier E., Géographies de l'Amérique Latine. PUR, p. 229-236.

Gouëset V., Demoraes F., Figueroa O., Le Roux G., Zioni S., 2014. Parcourir la métropole : pratiques de mobilité quotidienne et inégalités socio-territoriales. In Dureau F., Lulle T., Souchaud S., Contreras Y. Mobilités et changement urbain. Bogotá, Santiago et São Paulo. PUR, p. 265-302.

GRAB (Groupe de réflexion sur l'approche biographique), 1999. Biographies d'enquêtes. Bilan de 14 collectes biographiques. INED, IRD, Réseau Socio-Économie de l'habitat, Coll. Méthodes et savoirs $\mathrm{n}^{\mathrm{o}}$ 3, Paris, $340 \mathrm{p}$.

Grafmeyer Y., 2007. Le quartier des sociologues. In Authier J.-Y., Bacque M.-H., Guerin-Pace F. (dir.). Le quartier. Enjeux scientifiques, actions politiques et pratiques sociales. La Découverte, Coll. Recherches, Paris, p. 21-31.

Granovetter M., 1983. The strength of weak ties: a network theory revisited. Sociological Theory, Vol. 1, p. 201-233. 
Guérin-Pace F., 2003. Vers une typologie des territoires urbains de proximité. L'Espace géographique, $\mathrm{n}^{\circ}$ 4, p. 333-344.

Guérin-Pace F., 2007. Le quartier entre appartenance et attachement : une échelle identitaire ? In Authier J.-Y., Bacque M.-H., Guerin-Pace F. (dir.), Le quartier. Enjeux scientifiques, actions politiques et pratiques sociales. La Découverte, Coll. Recherches, Paris, p. 151-162.

Guerrien M., 2002. Pratiques et perceptions de l'espace urbain dans les quartiers populaires de Mexico. Cahier des Amériques Latines, $\mathrm{n}^{\circ} 39$, p. 129-154.

Guerrien M., 2004. L'enfance agitée d'une mégapole. Transition urbaine et fragmentation de l'espace dans la vallée de Mexico, Thèse de géographie, EHESS, Paris, 390 p.

Hiernaux-Nicolas D., Lindon A., 2003. Pratiques et stratégies résidentielles dans la Vallée de Chalco, périphérie de Mexico. Autrepart, n²5, p. 123-136.

Hilal M., Sencebé Y., 2002. Analyse des processus d'ancrage et des formes de territorialité des populations marquées par l'éclatement de leurs lieux de travail et de domicile à la périphérie des pôles urbains. Consultation de recherche « Mobilités et territoires urbains ». Note de synthèse, Dijon, CESAER, INRA ENESAD, $204 \mathrm{p}$.

IAU, 2015. Cahiers de l'IAU, $n^{\circ}$ 172, http://www.iau-idf.fr/savoir-faire/nos-travaux/amenagementet-territoires/les-bassins-de-vie.html

Imbert C., 2005. Les ancrages des habitants des villes nouvelles franciliennes : des bassins de vie en construction. Thèse de géographie, Université Paris 1, 390 p.

Imbert C., Brune A., Rozenholc C., 2011. Les villes nouvelles franciliennes, un exemple de périphéries en cours de maturation. Espace, Populations, Sociétés, $n^{\circ}$ 3, p. 591-602

Imbert C., Dubucs H., Dureau F., Giroud M., 2014. D'une métropole à l'autre. Pratiques urbaines et circulations dans l'espace européen, A. Colin, Recherches, Paris, 487 p.

INEGI, 2007. Encuesta Origen Destino 2007.

INEGI, 2010. XIII Censo general y conteo de Población y Vivienda 2010.

INEGI, 2012. Censos Económicos, 2009. Zonas Metropolitanas en los Estados Unidos Mexicanos. Censos Económicos 2009/Instituto Nacional de Estadística y Geografía, México, 3 volumes.

INEGI, 2013. DENUE 2013, Directorio Estadística Nacional de Unidades Económicas, México, http:// www3.inegi.org.mx/sistemas/mapa/denue/default.aspx.

INEGI, 2016a. Censos Económicos, 2014. http://www.inegi.org.mx/est/contenidos/Proyectos/ce/ ce2014/doc/tabulados.html.

INEGI, 2016b. Mortalidad. Conjunto de datos: defunciones por homicidios. http://www.inegi.org.mx/ sistemas/olap/proyectos/bd/continuas/mortalidad/defuncioneshom.asp?s=est

Jouffe Y., Caubel D., Fol S., Motte-Baumvol B., 2015. Faire face aux inégalités de mobilité. Cybergeo : European Journal of Geography. https://cybergeo.revues.org/26697

Lazo A., 2012. Entre le territoire de proximité et la mobilité quotidienne. Les ancrages et le territoire de proximité comme support et ressource pour les pratiques de mobilité des habitants de la ville de Santiago $d u$ Chili. Thèse de géographie, Université Toulouse 2, Pontifica Universidad Catolica de Chile, $442 \mathrm{p}$.

Le Bris E., 1981. Contenu géographique et contenu social de la notion de résidence. Quelques réflexions à partir de résultats d'enquêtes biographiques effectuées à Lomé, Togo et Accra, Ghana. Cahiers d'études africaines, vol. 21, n 81-83, p. 129-174. 
Lelévrier C., 2007. Mobilités et ancrages des familles en Île-de-France. Les changements de la rénovation urbaine. Informations sociales, $\mathrm{n}^{\circ} 141, \mathrm{CNAF}, \mathrm{p}$. 98-109.

Lelièvre E., Robette N., 2006. Les espaces de référence des individus : définir et mesurer les espaces de vie. In Bonvalet C., Lelievre E. (eds.), Publications choisies autour de l'enquête Biographies et entourage. Documents de travail, $\mathrm{n}^{\circ}$ 134, Paris, INED, 24 p.

Lessault D., Imbert C., 2013. Mobilité résidentielle et dynamique récente du peuplement urbain à Dakar (Sénégal). Cybergeo : European Journal of Geography https://cybergeo.revues.org/26146

Lévy J-P., 2009. Mobilités urbaines : des pratiques sociales aux évolutions territoriales. In Dureau F., Hily M-A., Les mondes de la mobilité. PUR, p. 105-136.

Lombard M., 2014. Constructing ordinary places: Place-making in urban informal settlements in Mexico. Progress in Planning, $\mathrm{n}^{\circ}$ 94, p. 1-53.

Lombard M., 2015. Lugarización y la construcción de asentamientos informales en México. Revista INVI, vol. 30, n 83, p. 117-146.

Marchal H., 2009. Identité du citadin. In Stébé J.-M., Marchal H. (dir.), Traité sur la ville. PUF, Paris, p. 399-460.

Massot M.-H. (dir.), 2010. Mobilités et modes de vie métropolitains : les intelligences du quotidien. L'œil d'or, Paris, 330 p.

Motte-Baumvol B., Bonin O., Nassi C.-D., Belton-Chevallier L., 2016. Barriers and (im)mobility in Rio de Janeiro. Urban Studies, vol. 53, n 14, p. 2956-2972.

OCIM-SIG (Observatorio de la Ciudad de México- Sistema de Información Geográfica). http:// www.ocim.azc.uam.mx/

Omhovère M., 2014. Vivre à Gambetta, l'ancrage local. Lorsque la proximité devient ressource. In Fol S., Miot Y., Vignal C., Mobilités résidentielles, territoires et politiques urbaines. Presses Universitaires du Septentrion, coll. Le regard sociologique », p. 229-248.

Ortar N., 2005. Le paradoxe de l'ancrage et de la mobilité en zone rurale et périurbaine. In Bonnet L., Bertrand L., Mobilités, habitat et identités, INED, coll. Documents de travail. http:// resohab.univ-paris1.fr/jclh05/article.php3?id_article=23

Paquette C., 2010. Mobilité quotidienne et accès à la ville des ménages périurbains dans l'agglomération de Mexico. Une lecture des liens entre pauvreté et mobilité. Revue Tiers Monde, $n^{\circ}$ 201/1, A. Colin, p. 157-175.

Pujol C., 2010. De l'ancrage mobile à l'inscription circulatoire : regards croisés sur les trajectoires citadines et citoyennes aux marges de Rosario, Argentine et de Montevideo, Uruguay. Thèse de géographie, Université Toulouse 2, $321 \mathrm{p}$.

Ramadier T., 2007. Mobilité quotidienne et attachement au quartier : une question de position? In Authier J.-Y., Bacqué M.-H., Guérin-Pace F. (dir.)., Le quartier. Enjeux scientifiques, actions politiques et pratiques sociales. La Découverte, Coll. Recherches, Paris, p. 127-138.

Rémy J., 1996. Mobilités et ancrages : vers une autre définition de la ville. In Hirschhorn M., Berthelot J.-M., Mobilités et ancrages. Vers un nouveau monde de spatialisation? L'Harmattan, Coll. Villes et entreprises, Paris, Montréal, p. 135-153.

Ribardière A., 2017. « Du Oaxaca à la ville de Mexico : accès aux ressources urbaines ». EchoGéo, même numéro. 
Ribardière A., Valette J.-F., 2013. Évolution de la division sociale de l'espace urbain à Mexico. Approche à partir des structures démographiques et de l'accès au diplôme, 1990-2010. Problèmes d'Amérique latine, $\mathrm{n}^{\circ}$ 90, p. 99-126.

Ribardière A., Valette J-F., 2014. La pauvreté urbaine à Mexico : une approche de la diversité des colonies populaires ", EchoGéo [En ligne], 30 | 2014, mis en ligne le 04 décembre 2014,. URL : http://echogeo.revues.org/14020 ; DOI : 10.4000/echogeo.14020

Ruiz Rivera N., Suárez M., Delgado J., 2016. Urban segregation and local retail environments. Evidence from Mexico City. Habitat International, $n^{\circ}$ 54, p. 58-64.

Salazar C., 2010. Dos sistemas de movilidad cotidiana en la Ciudad de México: domicilio-escuela y domicilio-trabajo. In Garza G., Schteingart M. (coord.), Los grandes problemas de México. Desarrollo urbano y regional. , El Colegio de México, vol. II, p. 511-550.

Salazar C., 2014. El Puño invisible' de la privatización. Territorios, n³ 30, p. 69-90.

Salazar C., Sobrino J., 2006. El área central de la ciudad de México: ¿Espacio de oportunidad laboral para la metrópoli? Communication au séminaire Réinvestir le "centre" : politique de requalification, transformations urbaines et pratiques citadines dans les quartiers centraux des grandes villes d'Amérique latine, Paris, 6-8 juin 2006, IRD/CREDAL-CNRS.

SEDESOL, CONAPO, INEGI, 2012. Delimitación de las zonas metropolitanas de México 2010, México.

Seguridad, Justicia y Paz, 2016. La violencia en los municipios de México 2015. Consejo Ciudadano para la Seguridad y Justicia Penal A.C., 80 p.

Stamm C., 2014. Expulsion et relocalisation du commerce de rue dans la métropole de Mexico. L'Espace Politique, $\mathrm{n}^{\circ} 22$, http://espacepolitique.revues.org/2974

Suárez M., Murata M., Delgado J., 2015. Why do the poor travel less? Urban structure, commuting, and economic informality in Mexico City? Urban Studies, p. 1-19.

Tomas F., 1995. Mexico : tous propriétaires ! Annales de la Recherche Urbaine, Paris, $\mathrm{n}^{\circ}$ 66, p. 72-80.

Valette J.-F., 2014. Mobilités et ancrages dans les quartiers populaires de la périphérie de Mexico : une approche de la maturation urbaine. Thèse de géographie, Université Paris 1, 955 p. https:// hal.archives-ouvertes.fr/tel-01134072

Valette J-F., 2015. L'hétérogénéité des colonias populares de la périphérie de Mexico : une approche des micro-divisions sociales dans le processus de maturation urbaine. L'Espace Géographique, $\mathrm{n}^{\circ} 4$, p. 289-306. https://www.cairn.info/revue-espace-geographique-2015-4-page-289.htm

\section{NOTES}

1. En 2017, la ZMVM compte 76 unités administratives : 16 délégations de la Ciudad de México (ex District Fédéral - DF), 59 municipalités de l'État de Mexico et une de l'État d'Hidalgo (SEDESOL et al, 2012).

2. De la traduction de colonia popular, terme générique utilisé au Mexique pour désigner un quartier populaire. La colonie est le vocable usité pour désigner un quartier.

3. En marge du cadre légal au moment de la fondation et/ou toujours à l'heure actuelle.

4. L'approche du quartier est ici celle de l'espace vécu et représenté par ses habitants (Di Méo, 1994 ; Tomas, 1995). L'absence de limites officielles de la colonie au Mexique n'altère pas une identification assez claire du territoire pour ses habitants. Si le quartier, dans la géographie française, reste un objet complexe et parfois vague, il s'agit d'avoir ici une approche simple pour 
faire référence à la portion de ville ou la configuration socio-spatiale dans laquelle s'inscrivent l'analyse sociologique urbaine et l'étude des comportements citadins (Grafmeyer, 2007 ; Marchal, 2009).

5. Voir entre autres les travaux de J.-Y. Authier (2001), J.-P. Lévy (2009), B. Florin et N. Semmoud (2010), S. Fol (2010), A. Lazo (2012) ou encore M. Omhovère (2014).

6. Sur cette combinaison, voir notamment les travaux de M. Hilal et Y. Sencébé (2002), C. Imbert (2005), N. Ortar (2005), C. Lelévrier (2007), N. Brevet (2008), A. Lazo (2012) ou J.-F. Valette (2014).

7. S'il n'est pas ici question de traiter directement l'appropriation du quartier par la dimension identitaire, voire de l'attachement, on peut renvoyer à la production notable en géographie et en psychologie sociale sur ces thèmes, comme dans les travaux de F. Guérin-Pace $(2003 ; 2007)$ ou T. Ramadier (2007).

8. La notion de bassin de vie, développée par les aménageurs français, sert à délimiter les territoires de l'accès aux logements, aux équipements et à l'emploi au-delà des limites administratives. Voir entre autres les travaux de C. Imbert (2005) et N. Brevet (2008), qui articulent cette notion avec celle d'ancrage, ou encore de S. Carpentier et al. sur le lien à établir entre bassins et espaces de vie (2010). La notion est aujourd'hui en France l'objet de débat, les hypothèses sous-jacentes aux découpages, les échelles, les objectifs et les méthodologies pouvant varier sensiblement selon les définitions qui en sont faites. Voir le $\mathrm{n}^{\circ} 172$ des Cahiers de l'IAU (IAU, 2015).

9. Dans les Suds, les travaux sur Bogotá, São Paulo et Santiago coordonnés par F. Dureau, ou encore ceux sur Dakar coordonnés par D. Lessault ont par exemple déjà mis en lumière de tels processus.

10. Réalisée sur près de 50000 logements dans 16 délégations du DF et 40 municipes de l'État de Mexico.

11. Pour les questions relatives aux mobilités quotidiennes, l'échantillon détaillé ne permet pas de géolocalisation plus précise que celle du municipe, tandis que les bases agrégées à des niveaux fins infra-municipaux ne décrivent pas ces variables.

12. Le maillage de l'enquête a découpé la zone métropolitaine en 156 districts, souvent plus fins que le découpage municipal, sauf pour 7 municipalités périphériques qui ont fait l'objet de regroupements.

13. Dans le texte, la référence au « local » ou à la «proximité » fait écho au maillage infra-urbain disponible relativement à la précision de l'information : de l'îlot/groupe d'îlots à la municipalité, en passant par le district et la colonie, le «local» correspond à l'espace observable «le plus proche " relativement à la résidence.

14. Les échantillons réunis n'ayant aucune vocation à être représentatifs d'un point de vue statistique, leur traitement «quantitatif» ne prend sens que par rapport à la richesse d'informations que les réponses sont susceptibles de révéler.

15. Les enquêtes ont été réalisées par l'auteur lors de 5 séjours de terrain de plus de 3 mois entre 2009 et 2011.

16. A savoir les rapports entretenus entre les individus et leur lieu de résidence (Authier, 2001, p. 134)

17. À l'exception notable de la marche. Sur ce dernier mode de déplacement, voir les travaux de P. López.

18. L'apparente immobilité de l'ancrage est considérée comme problématique, car elle impliquerait des pratiques centrées sur un espace local. Or, ces pratiques locales seraient ellesmêmes déstructurées par la valorisation de la mobilité - qui inciterait à dépasser un cadre spatial supposé étriqué. Par ailleurs, cette valorisation de la mobilité renforcerait aussi les inégalités d'accès aux ressources nécessaires à cette même mobilité (Fol, 2010, p. 27).

19. Fin 2016 dans la ZMVM, selon l'enquête nationale sur l'emploi (ENOE), le taux d'activité informelle - à savoir la part des actifs travaillant pour une unité économique dont les ressources 
sont celles du ménage et/ou dont l'activité n'a pas de situation identifiable - est de $30 \%$. Cette proportion s'élève à $50 \%$ pour le taux d'informalité au travail, c'est-à-dire la part de la population active en situation de vulnérabilité professionnelle, du fait de la nature de l'unité économique dans laquelle s'opère l'activité et/ou du fait que l'emploi n'est pas officiellement reconnu par l'employeur (et ne donne droit à aucune protection sociale).

20. Sur le thème du commerce à Mexico, voir les travaux de C. Stamm (2014), E. Duhau et A. Giglia (2007), et N. Ruiz-Rivera et al. (2016).

21. C'est-à-dire des contacts avec des groupes sociaux extérieurs à la communauté, moins nombreux et solides que les « liens forts » (amis, famille), mais plus efficaces, car plus diversifiés, notamment par rapport à la recherche d'emploi (Granovetter, 1983).

22. En 2014, le DF concentre encore 52 \% des unités économiques de la ZMVM, mais surtout $70 \%$ des emplois (plus d'un tiers rien que dans les 4 délégations centrales) et $80 \%$ de la valeur de la production (INEGI, 2016a).

23. Le DENUE prend en compte la plupart des activités économiques impliquant l'existence d'un établissement ou d'une entreprise, que l'emploi y soit reconnu ou non. Seuls sont exclus les taxis, les activités ambulantes, celles impliquant le montage-démontage quotidien d'un local, les activités de production agricole, celles développées par les organisations politiques, et l'emploi domestique au sein du foyer - limitant en cela la prise en compte d'une partie des activités informelles (INEGI, 2013).

24. Malgré l'apparente diversité et densité, une grande partie de ces petits commerces et ateliers peut montrer porte close (plusieurs jours par semaine, plusieurs semaines par mois). En effet, la régularité et l'intensité de l'activité ne sont pas systématiques. La durée de vie des entreprises est limitée, du fait des stratégies complexes et précaires des ménages-entrepreneurs.

25. La densité du tissu commercial présent dans les quartiers populaires de Mexico a une position particulière en comparaison de villes comme Lima ou Santiago du Chili (Avellaneda, Lazo, 2011 ; Paquette, 2010) où, l'offre commerciale, présentée comme moindre, voire insuffisante dans les périphéries pauvres, rendrait nécessaires les connexions très fortes aux autres territoires urbains, la recherche de ressources « imposant » un espace de vie très large dans la ville. En cela, les mobilités quotidiennes connectant les périphéries aux «zones centrales " seraient moins déterminantes à Mexico qu'ailleurs dans les dynamiques d'intégration ou d'exclusion sociale.

26. Au Mexique, le taux d'homicides était de 24 pour 100000 habitants au moment des enquêtes en 2011. En 2015, il est de 17 pour 100000 habitants (INEGI, 2016b), avec des scores allant de 7 à 19 dans les municipes étudiés (Seguridad Justicia y Paz, 2016).

\section{ABSTRACTS}

Within the illegal urban settlements, called colonias populares, on the outskirts of Mexico City, the population's anchorage does not seem obvious. This notion examines the circulation of individuals and their families in the different spatial and temporal scales of the living space. The analysis of the connections between people and their neighborhood highlights the role of residential context as a place of resources. From an empirical work and official surveys analysis, this paper aims to identify the different configurations of the places system on which inhabitants anchorages are built within the city. First, the links to their neighborhood in terms of practices allow to understand the space used in order to access to urban resources. Then, economic and social functionalization of the area is highlighted. In the end, crossing different practices of local 
and metropolitan areas allows the discovery of several types of links within the neighborhood and a variety of anchorages that fit into these places. Thus, the daily mobility underlines the urban resources existence, but also a relative intensity of movements on other scales, highlighting an integration process with the rest of the city.

$\mathrm{Au}$ sein des fronts pionniers urbains initialement irréguliers que constituent les colonies populaires de la périphérie de Mexico, l'ancrage des populations ne semble pas aller de soi. Cette notion interroge les circulations des individus et de leur entourage dans les différentes échelles spatiales et temporelles de l'espace de vie. L'analyse des rapports que tissent les habitants avec leur quartier permet de souligner le rôle du contexte résidentiel comme lieu de ressources. À partir d'un travail empirique et d'enquêtes officielles systématiques, il s'agit d'identifier les différentes configurations des systèmes de lieux sur lesquels se construisent les ancrages des habitants dans la ville. D'abord, les liens au quartier du point de vue des pratiques permettent d'appréhender l'espace mobilisé pour accéder aux ressources urbaines. Ensuite, une fonctionnalisation économique et sociale du quartier est mise en lumière. Au total, le croisement des différentes pratiques des espaces locaux et métropolitains autorise la mise au jour de plusieurs types de rapports au quartier et d'une diversité des ancrages qui s'inscrivent dans ces lieux. Les mobilités quotidiennes témoignent de la présence forte de ressources urbaines dans l'espace local, mais aussi d'une relative intensité des déplacements à d'autres échelles, allant dans le sens d'une intégration au reste de la ville.

Dentro de los frentes pioneros urbanos inicialmente irregulares que son las colonias populares de la periferia de la ciudad de México, el anclaje de las poblaciones no parece ser evidente. Esta noción examina la circulación de personas y sus familias en diferentes escalas espaciales y temporales del espacio de vida. El análisis de los vínculos entre los residentes y su barrio o colonia pueden destacar el papel del entorno residencial como lugar de recursos. A partir de un trabajo empírico y de encuestas oficiales, identificamos las diferentes configuraciones de los sistemas de lugares sobre los que se construyen los anclajes de los habitantes de la ciudad. En primer lugar, los vínculos con el barrio o la colonia, en términos de prácticas, permiten entender el espacio movilizado para acceder a los recursos urbanos. Después, se pone de relieve una funcionalización económica y social de la zona. Al final, cruzando diferentes prácticas de los espacios locales y metropolitanos permite el descubrimiento de varios tipos de vínculos con el barrio y una variedad de anclajes que se ajusten a estos lugares. La movilidad cotidiana subraya la existencia de los recursos urbanos en el espacio local, sino también una intensidad relativa de los movimientos en otras escalas, destacando una integración con el resto de la ciudad.

\section{INDEX}

Keywords: anchorages, daily mobility, low income neighborhood, Mexico City, urban resources Mots-clés: ancrage, Mexico, mobilité quotidienne, quartier populaire, ressource urbaine Palabras claves: anclajes, colonias populares, Zona metropolitana de la Ciudad de México, movilidad cotidiana, recursos urbanos

Subjects: Sur le Champ - Sur le Terrain 


\section{AUTHOR}

\section{JEAN-FRANÇOIS VALETTE}

Jean-François Valette, jean-francois.valette02@univ-paris8.fr, est Maître de Conférences à l'Université Paris 8 Vincennes-Saint-Denis, membre de l'UMR LADYSS et chercheur associé à l'UMR Prodig. Il a publié récemment :

- Valette J.-F., 2015. L'hétérogénéité des colonias populares de la périphérie de Mexico : une approche des micro-divisions sociales dans le processus de maturation urbaine. L'Espace Géographique, $\mathrm{n}^{\circ}$ 4, p. 289-306.

- Ribardière A., Valette J.-F., 2014. La pauvreté urbaine à Mexico : une approche de la diversité des colonies populaires, EchoGéo [En ligne], 30 | 2014, mis en ligne le 04 décembre 2014, http:// echogeo.revues.org/14020 ; DOI : 10.4000/echogeo.14020

- Valette J.-F., 2013. Le rôle des organisations sociales dans la régularisation des quartiers illégaux de la périphérie de Mexico. Autrepart, n66, p. 43-60. 